\title{
Sharp ill-posedness and well-posedness results for the KdV-Burgers equation: the real line case
}

\author{
LuC Molinet And Stéphane Vento
}

\begin{abstract}
We complete the known results on the Cauchy problem in Sobolev spaces for the KdV-Burgers equation by proving that this equation is well-posed in $H^{-1}(\mathbb{R})$ with a solution-map that is analytic from $H^{-1}(\mathbb{R})$ to $C\left([0, T] ; H^{-1}(\mathbb{R})\right)$ whereas it is ill-posed in $H^{s}(\mathbb{R})$, as soon as $s<-1$, in the sense that the flowmap $u_{0} \mapsto u(t)$ cannot be continuous from $H^{s}(\mathbb{R})$ to even $\mathcal{D}^{\prime}(\mathbb{R})$ at any fixed $t>0$ small enough. As far as we know, this is the first result of this type for a dispersive-dissipative equation. The framework we develop here should be useful to prove similar results for other dispersive-dissipative models.
\end{abstract}

Mathematics Subject Classification (2010): 35E15 (primary); 35M11, 35Q53, 35Q60 (secondary).

\section{Introduction and main results}

The aim of this paper is to establish positive and negative optimal results on the local Cauchy problem in Sobolev spaces for the Korteweg-de Vries-Burgers (KdVB) equation posed on the real line:

$$
u_{t}+u_{x x x}-u_{x x}+u u_{x}=0
$$

where $u=u(t, x)$ is a real-valued function.

This equation has been derived as an asymptotic model for the propagation of weakly nonlinear dispersive long waves in some physical contexts when dissipative effects occur (see [17]). It thus seems natural to compare the well-posedness results on the Cauchy problem for the KdV-B equation with those for the Korteweg-deVries $(\mathrm{KdV})$ equation

$$
u_{t}+u_{x x x}+u u_{x}=0
$$

that corresponds to the case where the dissipative effects are negligible, and for the dissipative Burgers $(\mathrm{dB})$ equation

$$
u_{t}-u_{x x}+u u_{x}=0
$$

that corresponds to the case where the dissipative effect are dominant.

L. M. was partially supported by the ANR project "Equa-Disp".

Received December 3, 2009; accepted in revised form May 3, 2010. 
To make this comparison more transparent it is convenient to define different notions of well-posedness (and consequently ill-posedness) related to the smoothness of the flow-map (see in the same spirit $[7,12]$ ).

Throughout this paper we shall say that a Cauchy problem is (locally) $C^{0}$-wellposed in some normed function space $X$ if, for any initial data $u_{0} \in X$, there exist a radius $R>0$, a time $T>0$ and a unique solution $u$, belonging to some space-time function space continuously embedded in $C([0, T] ; X)$, such that for any $t \in[0, T]$ the map $u_{0} \mapsto u(t)$ is continuous from the ball of $X$ centered at $u_{0}$ with radius $R$ into $X$. If the map $u_{0} \mapsto u(t)$ is of class $C^{k}, k \in \mathbb{N} \cup\{\infty\}$, (respectively analytic) we will say that the Cauchy is $C^{k}$-well-posed (respectively analytically well-posed). Finally a Cauchy problem will be said to be $C^{k}$-ill-posed, $k \in \mathbb{N} \cup\{\infty\}$, if it is not $C^{k}$-well-posed.

For the $\mathrm{KdV}$ equation on the line the situation is as follows: it is analytically well-posed in $H^{-3 / 4}(\mathbb{R})\left(c f\right.$. $[10,13,14]$ for the limit case) and $C^{3}$-ill-posed below this index ${ }^{1}(c f .[4])$. On the other hand the results for the dissipative Burgers equation are much clear. Indeed this equation is known to be analytically well-posed in $H^{s}(\mathbb{R})$ for $s \geq-1 / 2\left(c f\right.$. [1,6] for the limit case) and $C^{0}$-ill-posed in $H^{s}$ for $s<-1 / 2$ (cf. [6]). At this stage it is interesting to notice that the critical Sobolev exponents obtained by scaling considerations are respectively $-3 / 2$ for the $\mathrm{KdV}$ equation and $-1 / 2$ for the dissipative Burgers equation. Hence for the $\mathrm{KdV}$ equation there is an important gap between this critical exponent and the best exponent obtained for well-posedness.

Now, concerning the KdV-B equation, Molinet and Ribaud [16] proved that this equation is analytically well-posed in $H^{s}(\mathbb{R})$ as soon as $s>-1$. They also established that the index -1 is critical for $C^{2}$-well-posedness. The surprising part of this result was that, according to the above results, the $C^{\infty}$ critical index $s_{c}^{\infty}(\mathrm{KdV}-\mathrm{B})=-1$ was lower than that of the $\mathrm{KdV}$ equation $s_{c}^{\infty}(\mathrm{KdV})=-3 / 4$ and also lower than the $C^{\infty}$ index $s_{c}^{\infty}(\mathrm{dB})=-1 / 2$ of the dissipative Burgers equation.

In this paper we want in some sense to complete this study by proving that the $\mathrm{KdV}-\mathrm{B}$ equation is analytically well-posed in $H^{-1}(\mathbb{R})$ and $C^{0}$-ill-posed in $H^{s}(\mathbb{R})$ for $s<-1$ in the sense that the flow-map defined on $H^{-1}(\mathbb{R})$ is not continuous for the topology induced by $H^{s}, s<-1$, with values even in $\mathcal{D}^{\prime}(\mathbb{R})$. It is worth emphasizing that the critical index $s_{c}^{0}=-1$ is still far away from the critical index $s_{c}=-3 / 2$ given by the scaling symmetry of the KdV equation. We believe that this result strongly suggest that the $\mathrm{KdV}$ equation should also be $C^{0}$-ill-posed in $H^{s}(\mathbb{R})$ for $s<-1$.

To reach the critical Sobolev space $H^{-1}(\mathbb{R})$ we adapt the refinement of Bourgain's spaces that appeared in [20] and [19] to the framework developed in [16]. One of the main difficulties is related to the choice of the extension for negative times of the Duhamel operator (see the discussion at the beginning of Section 4). The approach we develop here to overcome this difficulty should be useful to prove optimal results for other dispersive-dissipative models. The ill-posedness result is

${ }^{1}$ See also [5] where it is proven that the solution-map is not even uniformly continuous on bounded sets below this index. 
due to a high to low frequency cascade phenomenon that was first observed in [2] for a quadratic Schrödinger equation.

At this stage it is worth noticing that, using integrability theory, it was recently proved in [12] that the flow-map of the KdV equation can be uniquely continuously extended in $H^{-1}(\mathbb{T})$. Therefore, on the torus, $\mathrm{KdV}$ is $C^{0}$-well-posed in $H^{-1}$ if one takes as uniqueness class, the class of strong limit in $C\left([0, T] ; H^{-1}(\mathbb{T})\right)$ of smooth solutions. In the present work we use in a crucial way the global Kato smoothing effect that does not hold on the torus. However, in a forthcoming paper ( [15]) we will show how one can modify the approach developed here to prove that the same results hold on the torus, i.e., analytic well-posedness in $H^{-1}(\mathbb{T})$ and $C^{0}$-illposedness in $H^{s}(\mathbb{T})$ for $s<-1$. In view of the result of Kappeler and Topalov for $\mathrm{KdV}$ it thus appears that, at least on the torus, even if the dissipation part of the KdV-B equation (it is important to notice that the dissipative term $-u_{x x}$ is of lower order than the dispersive one $u_{x x x}$ ) allows to lower the $C^{\infty}$ critical index with respect to the $\mathrm{KdV}$ equation, it does not permit to improve the $C^{0}$ critical index.

Our results can be summarized as follows:

Theorem 1.1. The Cauchy problem associated to (1.1) is locally analytically wellposed in $H^{-1}(\mathbb{R})$. Moreover, at every point $u_{0} \in H^{-1}(\mathbb{R})$ there exist $T=T\left(u_{0}\right)>$ 0 and $R=R\left(u_{0}\right)>0$ such that the solution-map $u_{0} \mapsto u$ is analytic from the ball centered at $u_{0}$ with radius $R$ of $H^{-1}(\mathbb{R})$ into $C\left([0, T] ; H^{-1}(\mathbb{R})\right)$. Finally, the solution $u$ can be extended for all positive times and belongs to $C\left(\mathbb{R}_{+}^{*} ; H^{\infty}(\mathbb{R})\right)$.

Theorem 1.2. The Cauchy problem associated to (1.1) is ill-posed in $H^{s}(\mathbb{R})$ for $s<-1$ in the following sense: there exists $T>0$ such that for any $0<t<T$, the flow-map $u_{0} \mapsto u(t)$ constructed in Theorem 1.1 is discontinuous at the origin from $H^{-1}(\mathbb{R})$ endowed with the topology induced by $H^{s}(\mathbb{R})$ into $\mathcal{D}^{\prime}(\mathbb{R})$.

\section{Ill-posedness}

The ill-posedness result can be viewed as an application of a general result proved in [2]. Roughly speaking this general ill-posedness result requires the following two ingredients:

1. The equation is analytically well-posed until some index $s_{c}^{\infty}$ with a solution-map that is also analytic.

2. Below this index one iteration of the Picard scheme is not continuous. The discontinuity should be driven by high frequency interactions that blow up in frequencies of order less than or equal to one.

The first ingredient is given by Theorem 1.1 whereas the second one has been derived in [16] where the discontinuity of the second iteration of the Picard scheme in $H^{s}(\mathbb{R})$ and $H^{s}(\mathbb{T})$ for $s<-1$ is established.

However, due to the nature of the equation, our result is a little better than that given by the general theory developed in [2]. Indeed, we will be able to prove the 
discontinuity of the flow-map $u_{0} \mapsto u(t)$ for any fixed $t>0$ less than some $T>0$ and not only of the solution-map $u_{0} \mapsto u$. Therefore for sake of completeness we will prove the result by hand here.

Let us first recall the counter example constructed in [16] that we renormalize here in $H^{-1}(\mathbb{R})$. We define the sequence of initial data $\left\{\phi_{N}\right\}_{N \geq 1}$ by

$$
\hat{\phi}_{N}=N\left(\chi_{I_{N}}(\xi)+\chi_{I_{N}}(-\xi)\right),
$$

where $I_{N}=[N, N+2]$ and $\hat{\phi}_{N}$ denotes the space Fourier transform of $\phi_{N}$. Note that $\left\|\phi_{N}\right\|_{H^{-1}(\mathbb{R})} \sim 1$ and $\phi_{N} \rightarrow 0$ in $H^{s}(\mathbb{R})$ for $s<-1$. This sequence yields a counter example to the continuity of the second iteration of the Picard Scheme in $H^{s}(\mathbb{R}), s<-1$, that is given by

$$
A_{2}(t, h, h)=\int_{0}^{t} S\left(t-t^{\prime}\right) \partial_{x}\left[S\left(t^{\prime}\right) h\right]^{2} d t^{\prime}
$$

where $S$ is the semi group associated to the linear part of (1.1) (see (3.2)). Indeed, computing the space Fourier transform we get

$$
\begin{aligned}
\mathcal{F}_{x}\left(A_{2}\left(t, \phi_{N}, \phi_{N}\right)\right)(\xi)= & \int_{\mathbb{R}} e^{-t \xi^{2}} e^{i t \xi^{3}} \hat{\phi}_{N}\left(\xi_{1}\right) \hat{\phi}_{N}\left(\xi-\xi_{1}\right) \\
& (i \xi) \int_{0}^{t} e^{-\left(\xi_{1}^{2}+\left(\xi-\xi_{1}\right)^{2}-\xi^{2}\right) t^{\prime}} e^{i\left(\xi_{1}^{3}+\left(\xi-\xi_{1}\right)^{3}-\xi^{3}\right) t^{\prime}} d t^{\prime} d \xi_{1} \\
= & (i \xi) e^{i t \xi^{3}} e^{-t \xi^{2}} \int_{\mathbb{R}} \hat{\phi}_{N}\left(\xi_{1}\right) \hat{\phi}_{N}\left(\xi-\xi_{1}\right) \\
& \frac{e^{-\left(\xi_{1}^{2}+\left(\xi-\xi_{1}\right)^{2}-\xi^{2}\right) t} e^{i 3 \xi \xi_{1}\left(\xi-\xi_{1}\right) t}-1}{-2 \xi_{1}\left(\xi-\xi_{1}\right)+i 3 \xi \xi_{1}\left(\xi-\xi_{1}\right)} d \xi_{1},
\end{aligned}
$$

so that

$$
\begin{aligned}
\left\|A_{2}\left(t, \phi_{N}, \phi_{N}\right)\right\|_{H^{s}}^{2} & \geq \int_{-1 / 2}^{1 / 2}\left(1+|\xi|^{2}\right)^{s}\left|\mathcal{F}_{x}\left(A_{2}\left(t, \phi_{N}, \phi_{N}\right)\right)(\xi)\right|^{2} d \xi \\
& =N^{4} \int_{-1 / 2}^{1 / 2}\left(1+|\xi|^{2}\right)^{s}|\xi|^{2} \\
& \quad\left|\int_{K_{\xi}} \frac{e^{-\left(\xi_{1}^{2}+\left(\xi-\xi_{1}\right)^{2}\right) t} e^{i 3 \xi \xi_{1}\left(\xi-\xi_{1}\right) t}-e^{-\xi^{2} t}}{-2 \xi_{1}\left(\xi-\xi_{1}\right)+i 3 \xi \xi_{1}\left(\xi-\xi_{1}\right)} d \xi_{1}\right|^{2} d \xi,
\end{aligned}
$$

where

$$
K_{\xi}=\left\{\xi_{1} / \xi-\xi_{1} \in I_{N}, \xi_{1} \in-I_{N}\right\} \cup\left\{\xi_{1} / \xi_{1} \in I_{N}, \xi-\xi_{1} \in-I_{N}\right\} .
$$

Note that for any $\xi \in[-1 / 2,1 / 2]$, one has $\operatorname{mes}\left(K_{\xi}\right) \geq 1$ and

$$
\left\{\begin{array}{rl}
3 \xi_{1}\left(\xi-\xi_{1}\right) & \sim N^{2} \\
2 \xi_{1}\left(\xi-\xi_{1}\right) & \sim N^{2}
\end{array}, \quad \forall \xi_{1} \in K_{\xi} .\right.
$$


Therefore, fixing $0<t<1$ we have

$$
\mathcal{R} e\left(e^{-\left(\xi_{1}^{2}+\left(\xi-\xi_{1}\right)^{2}\right) t} e^{i 3 \xi \xi_{1}\left(\xi-\xi_{1}\right) t}-e^{-\xi^{2} t}\right) \leq-e^{-t / 4}+e^{-2(N+2)^{2} t},
$$

which leads for $N=N(t)>0$ large enough to

$$
\left|\int_{K_{\xi}} \frac{e^{-\left(\xi_{1}^{2}+\left(\xi-\xi_{1}\right)^{2}\right) t} e^{i 3 \xi \xi_{1}\left(\xi-\xi_{1}\right) t}-e^{-\xi^{2} t}}{-2 \xi_{1}\left(\xi-\xi_{1}\right)+i 3 \xi \xi_{1}\left(\xi-\xi_{1}\right)} d \xi_{1}\right| \geq C \frac{e^{-t / 4}}{N^{2}}
$$

and thus

$$
\left\|A_{2}\left(t, \phi_{N}, \phi_{N}\right)\right\|_{H^{s}}^{2} \geq C e^{-t / 4} \geq C_{0}
$$

for some positive constant $C_{0}$. Since $\phi_{N} \rightarrow 0$ in $H^{s}(\mathbb{R})$, for $s<-1$, this ensures that, for any fixed $t>0$, the map $u_{0} \mapsto A_{2}\left(t, u_{0}, u_{0}\right)$ is not continuous at the origin from $H^{s}(\mathbb{R})$ into $H^{s}(\mathbb{R})$. Actually since one can replace $A_{2}\left(t_{N}, \phi_{N}, \phi_{N}\right)$ by its projection on the low frequencies in (2.2) we also get the discontinuity from $H^{s}(\mathbb{R})$ into $\mathcal{D}^{\prime}(\mathbb{R})$.

Now, we will use that $A_{2}\left(t, \phi_{N}, \phi_{N}\right)$ is of order of one in $H^{s}(\mathbb{R})$ to prove that somehow $A_{2}\left(t, \varepsilon \phi_{N}, \varepsilon \phi_{N}\right)$ is the main contribution to $u\left(t, \varepsilon \phi_{N}\right)$ in $H^{s}(\mathbb{R})$ as soon as $s<-1, \varepsilon>0$ is small and $N$ is large enough. The discontinuity of $u_{0} \mapsto u(t)$ will then follow from that of $u_{0} \mapsto A_{2}\left(t, u_{0}, u_{0}\right)$.

According to Theorem 1.1 there exist $T>0$ and $\varepsilon_{0}>0$ such that for any $|\varepsilon| \leq \varepsilon_{0}$, any $\|h\|_{H^{-1}(\mathbb{R})} \leq 1$ and $0 \leq t \leq T$,

$$
u(t, \varepsilon h)=\varepsilon S(t) h+\sum_{k=2}^{+\infty} \varepsilon^{k} A_{k}\left(t, h^{k}\right)
$$

where $h^{k}:=(h, \ldots, h), h^{k} \mapsto A_{k}\left(t, h^{k}\right)$ is a $k$-linear continuous map from $H^{-1}(\mathbb{R})^{k}$ into $C\left([0, T] ; H^{-1}(\mathbb{R})\right)$ and the series converges absolutely in $C\left([0, T] ; H^{-1}(\mathbb{R})\right)$. In particular,

$$
u\left(t, \varepsilon \phi_{N}\right)-\varepsilon^{2} A_{2}\left(t, \phi_{N}, \phi_{N}\right)=\varepsilon S(t) \phi_{N}+\sum_{k=3}^{+\infty} \varepsilon^{k} A_{k}\left(t, \phi_{N}^{k}\right)
$$

On the other hand, $\left\|S(t) \phi_{N}\right\|_{H^{s}(\mathbb{R})} \leq\left\|\phi_{N}\right\|_{H^{s}(\mathbb{R})} \sim N^{1+s}$ and

$$
\left\|\sum_{k=3}^{\infty} \varepsilon^{k} A_{k}\left(t, \phi_{N}^{k}\right)\right\|_{H^{-1}} \leq\left(\frac{\varepsilon}{\varepsilon_{0}}\right)^{3} \sum_{k=3}^{\infty} \varepsilon_{0}^{k}\left\|A_{k}\left(t, \phi_{N}^{k}\right)\right\|_{H^{-1}} \leq C \varepsilon^{3} .
$$

Hence, for $s<-1$,

$$
\sup _{t \in[0, T]}\left\|u\left(t, \varepsilon \phi_{N}\right)-\varepsilon^{2} A_{2}\left(t, \phi_{N}, \phi_{N}\right)\right\|_{H^{s}(\mathbb{R})} \leq C \varepsilon^{3}+O\left(N^{1+s}\right) .
$$


In view of (2.2) this ensures that, fixing $0<t<1$ and taking $\varepsilon$ small enough and $N$ large enough, $\varepsilon^{2} A_{2}\left(t, \phi_{N}, \phi_{N}\right)$ is a "good" approximation of $u\left(t, \varepsilon \phi_{N}\right)$. In particular, taking $\varepsilon \leq C_{0} C^{-1} / 4$ we get

$$
\left\|u\left(t, \varepsilon \phi_{N}\right)\right\|_{H^{s}(\mathbb{R})} \geq C_{0} \varepsilon^{2} / 2+O\left(N^{1+s}\right) .
$$

Since $u(t, 0) \equiv 0$ and $\phi_{N} \rightarrow 0$ in $H^{s}(\mathbb{R})$ for $s<-1$ this leads to the discontinuity of the flow-map from $H^{s}(\mathbb{R})$ into $H^{s}(\mathbb{R})$ at the origin by letting $N$ tend to infinity. Again since one can replace $u\left(t, \varepsilon \phi_{N}\right)$ by its projection on the low frequencies in the last inequality, the discontinuity of the flow-map from $H^{s}(\mathbb{R})$ into $\mathcal{D}^{\prime}(\mathbb{R})$ follows. Finally, it is worth noticing that since $\phi_{N} \rightarrow 0$ in $H^{-1}(\mathbb{R})$ we also get that $u_{0} \mapsto u\left(t, u_{0}\right)$ is discontinuous from $H^{-1}(\mathbb{R})$ equipped with its weak topology with values even in $\mathcal{D}^{\prime}(\mathbb{R})$.

\section{Resolution space}

In this section we introduce some notation and we define our functional framework.

For $A, B>0, A \lesssim B$ means that there exists $c>0$ such that $A \leq c B$. When $c$ is a small constant we use $A \ll B$. We write $A \sim B$ to denote the statement that $A \lesssim B \lesssim A$. For $u=u(t, x) \in \mathcal{S}^{\prime}\left(\mathbb{R}^{2}\right.$ ), we denote by $\widehat{u}$ (or $\mathcal{F}_{x} u$ ) its Fourier transform in space, and by $\tilde{u}$ (or $\mathcal{F} u$ ) the space-time Fourier transform of $u$. We consider the usual Lebesgue spaces $L^{p}, L_{x}^{p} L_{t}^{q}$ and abbreviate $L_{x}^{p} L_{t}^{p}$ as $L^{p}$. Let us define the Japanese bracket $\langle x\rangle=\left(1+|x|^{2}\right)^{1 / 2}$ so that the standard nonhomogeneous Sobolev spaces are endowed with the norm $\|f\|_{H^{s}}=\left\|\langle\nabla\rangle^{s} f\right\|_{L^{2}}$.

We also need a Littlewood-Paley analysis. Let $\eta \in C_{0}^{\infty}(\mathbb{R})$ be such that $\eta \geq 0$, $\operatorname{supp} \eta \subset[-2,2], \eta \equiv 1$ on $[-1,1]$. We next define $\varphi(\xi)=\eta(\xi)-\eta(2 \xi)$. Any summation over capitalized variables such as $N, L$ is presumed to be dyadic, i.e., these variables range over numbers of the form $2^{\ell}, \ell \in \mathbb{Z}$. We set $\varphi_{N}(\xi)=\varphi(\xi / N)$ and define the operator $P_{N}$ by $\mathcal{F}\left(P_{N} u\right)=\varphi_{N} \widehat{u}$. We introduce $\psi_{L}(\tau, \xi)=\varphi_{L}(\tau-$ $\left.\xi^{3}\right)$ and, for any $u \in \mathcal{S}^{\prime}\left(\mathbb{R}^{2}\right)$,

$$
\mathcal{F}_{x}\left(P_{N} u(t)\right)(\xi)=\varphi_{N}(\xi) \hat{u}(t, \xi), \quad \mathcal{F}\left(Q_{L} u\right)(\tau, \xi)=\psi_{L}(\tau, \xi) \tilde{u}(\tau, \xi) .
$$

Roughly speaking, the operator $P_{N}$ localizes in the annulus $\{|\xi| \sim N\}$ whereas $Q_{L}$ localizes in the region $\left\{\left|\tau-\xi^{3}\right| \sim L\right\}$.

Furthermore we define more general projections $P \lesssim_{\lesssim N}=\sum_{N_{1} \lesssim N} P_{N_{1}}, Q_{\gg L}=$ $\sum_{L_{1} \gg L} Q_{L_{1}}$ etc.

Let $e^{-t \partial_{x x x}}$ be the propagator associated to the Airy equation and define the two-parameter linear operator $W$ by

$$
\mathcal{F}_{x}\left(W\left(t, t^{\prime}\right) \phi\right)(\xi)=\exp \left(i t \xi^{3}-\left|t^{\prime}\right| \xi^{2}\right) \hat{\phi}(\xi), \quad t \in \mathbb{R} .
$$

The operator $W: t \mapsto W(t, t)$ is clearly an extension to $\mathbb{R}$ of the linear semi group $S(\cdot)$ associated with (1.1) that is given by

$$
\mathcal{F}_{x}(S(t) \phi)(\xi)=\exp \left(i t \xi^{3}-t \xi^{2}\right) \hat{\phi}(\xi), \quad t \in \mathbb{R}_{+} .
$$


We will mainly work on the integral formulation of (1.1):

$$
u(t)=S(t) u_{0}-\frac{1}{2} \int_{0}^{t} S\left(t-t^{\prime}\right) \partial_{x} u^{2}\left(t^{\prime}\right) d t^{\prime}, \quad t \in \mathbb{R}_{+} .
$$

Actually, to prove the local existence result, we will apply a fixed-point argument to the following extension of (3.3) (see Section 4 for some explanations on this choice).

$$
\begin{array}{r}
u(t)=\eta(t)\left[W(t) u_{0}-\frac{1}{2} \chi_{\mathbb{R}_{+}}(t) \int_{0}^{t} W\left(t-t^{\prime}, t-t^{\prime}\right) \partial_{x} u^{2}\left(t^{\prime}\right) d t^{\prime}\right. \\
\left.-\frac{1}{2} \chi_{\mathbb{R}_{-}}(t) \int_{0}^{t} W\left(t-t^{\prime}, t+t^{\prime}\right) \partial_{x} u^{2}\left(t^{\prime}\right) d t^{\prime}\right] .
\end{array}
$$

It is clear that if $u$ solves (3.4) then $u$ is a solution of (3.3) on [0,T], T<1.

In [16], the authors performed the iteration process in the space $X^{s, b}$ equipped with the norm

$$
\|u\|_{X^{s, b}}=\left\|\left\langle i\left(\tau-\xi^{3}\right)+\xi^{2}\right\rangle^{b}\langle\xi\rangle^{s} \widetilde{u}\right\|_{L^{2}}
$$

which takes advantage of the mixed dispersive-dissipative part of the equation. In order to handle the endpoint index $s=-1$ without encountering logarithmic divergence, we will rather work in its Besov version $X^{s, b, q}$ (with $q=1$ ) defined as the weak closure of the test functions that are uniformly bounded by the norm

$$
\|u\|_{X^{s, b, q}}=\left(\sum_{N}\left[\sum_{L}\langle N\rangle^{s q}\left\langle L+N^{2}\right\rangle^{b q}\left\|P_{N} Q_{L} u\right\|_{L_{x t}^{2}}^{q}\right]^{2 / q}\right)^{1 / 2} .
$$

This Besov refinement, which usually provides suitable control for the nonlinear terms, is not sufficient here to get the desired bound especially in the high-high regime, where the nonlinearity makes interact two components of the solution $u$ with the same high frequency. To handle these divergences, inspired by [19], we introduce, for $b \in\left\{\frac{1}{2},-\frac{1}{2}\right\}$, the space $Y^{s, b}$ endowed with the norm

$$
\|u\|_{Y^{s, b}}=\left(\sum_{N}\left[\langle N\rangle^{s}\left\|\mathcal{F}^{-1}\left[\left(i\left(\tau-\xi^{3}\right)+\xi^{2}+1\right)^{b+1 / 2} \varphi_{N} \tilde{u}\right]\right\|_{L_{t}^{1} L_{x}^{2}}\right]^{2}\right)^{1 / 2},
$$

so that

$$
\|u\|_{Y^{-1, \frac{1}{2}}} \sim\left(\sum_{N}\left[\langle N\rangle^{-1}\left\|\left(\partial_{t}+\partial_{x x x}-\partial_{x x}+I\right) P_{N} u\right\|_{L_{t}^{1} L_{x}^{2}}\right]^{2}\right)^{1 / 2} .
$$

We next form the resolution space $\mathcal{S}^{s}=X^{s, \frac{1}{2}, 1}+Y^{s, \frac{1}{2}}$, and the "nonlinear space" $\mathcal{N}^{s}=X^{s,-\frac{1}{2}, 1}+Y^{s,-\frac{1}{2}}$ in the usual way:

$$
\|u\|_{X+Y}=\inf \left\{\left\|u_{1}\right\|_{X}+\left\|u_{2}\right\|_{Y}: u_{1} \in X, u_{2} \in Y, u=u_{1}+u_{2}\right\} .
$$

In the rest of this section we study some basic properties of the function space $\mathcal{S}^{-1}$. 
Lemma 3.1. For any $\phi \in L^{2}$,

$$
\left(\sum_{L}\left[L^{1 / 2}\left\|Q_{L}\left(e^{-t \partial_{x x x}} \phi\right)\right\|_{L^{2}}\right]^{2}\right)^{1 / 2} \lesssim\|\phi\|_{L^{2}} .
$$

Proof. From the Plancherel theorem, we have

$$
\left(\sum_{L}\left[L^{1 / 2}\left\|Q_{L}\left(e^{-t \partial_{x x x}} \phi\right)\right\|_{L^{2}}\right]^{2}\right)^{1 / 2} \sim\left\|\left|\tau-\xi^{3}\right|^{1 / 2} \mathcal{F}\left(e^{-t \partial_{x x x}} \phi\right)\right\|_{L^{2}} .
$$

Moreover if we set $\eta_{T}(t)=\eta(t / T)$ for $T>0$, then

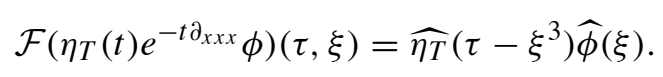

Thus we obtain with the changes of variables $\tau-\xi^{3} \rightarrow \tau^{\prime}$ and $T \tau^{\prime} \rightarrow \sigma$ that

$$
\left\|\left|\tau-\xi^{3}\right|^{1 / 2} \mathcal{F}\left(\eta_{T}(t) e^{-t \partial_{x x x}} \phi\right)\right\|_{L^{2}} \lesssim\|\phi\|_{L^{2}}\left\|\left|\tau^{\prime}\right|^{1 / 2} T \widehat{\eta}\left(T \tau^{\prime}\right)\right\|_{L_{\tau^{\prime}}^{2}} \lesssim\|\phi\|_{L^{2}} .
$$

Taking the limit $T \rightarrow \infty$, this completes the proof.

\section{Lemma 3.2.}

1. For each dyadic $N$, we have

$$
\left\|\left(\partial_{t}+\partial_{x x x}\right) P_{N} u\right\|_{L_{t}^{1} L_{x}^{2}} \lesssim\left\|P_{N} u\right\|_{Y^{0, \frac{1}{2}}} .
$$

2. For all $u \in \mathcal{S}^{-1}$,

$$
\|u\|_{L_{x t}^{2}} \lesssim\|u\|_{\mathcal{S}^{-1}}
$$

3. For all $u \in \mathcal{S}^{0}$,

$$
\left(\sum_{L}\left[L^{1 / 2}\left\|Q_{L} u\right\|_{L^{2}}\right]^{2}\right)^{1 / 2} \lesssim\|u\|_{\mathcal{S}^{0}}
$$

\section{Proof.}

1. From the definition of $Y^{0, \frac{1}{2}}$, the right-hand side of (3.5) can be rewritten as

$$
\left\|P_{N} u\right\|_{Y^{0, \frac{1}{2}}}=\left\|\left(\partial_{t}+\partial_{x x x}-\partial_{x x}+I\right) P_{N} u\right\|_{L_{t}^{1} L_{x}^{2}} .
$$

Thus, by the triangle inequality, it is equivalent to prove (3.5) with $\partial_{t}+\partial_{x x x}$ replaced by $I-\partial_{x x}$. Using the Plancherel theorem as well as the Young and Hölder inequalities, we get the

$$
\begin{aligned}
& \left\|\left(I-\partial_{x x}\right) P_{N} u\right\|_{L_{t}^{1} L_{x}^{2}} \\
& \quad \lesssim\left\|\mathcal{F}_{t}^{-1}\left(\frac{\xi^{2}+1}{i\left(\tau-\xi^{3}\right)+\xi^{2}+1}\left(i\left(\tau-\xi^{3}\right)+\xi^{2}+1\right) \varphi_{N} \tilde{u}\right)\right\|_{L_{t}^{1} L_{\xi}^{2}} .
\end{aligned}
$$


In the sequel, it will be convenient to write $\varphi_{N}$ for $\varphi_{N / 2}+\varphi_{N}+\varphi_{2 N}$. With this slight abuse of notation, we obtain

$$
\begin{aligned}
& \left\|\left(I-\partial_{x x}\right) P_{N} u\right\|_{L_{t}^{1} L_{x}^{2}} \\
& \quad \lesssim\left\|\mathcal{F}_{t}^{-1}\left(\frac{\varphi_{N}(\xi)\left(\xi^{2}+1\right)}{i\left(\tau-\xi^{3}\right)+\xi^{2}+1}\right)\right\|_{L_{t}^{1} L_{\xi}^{\infty}}\left\|\left(\partial_{t}+\partial_{x x x}-\partial_{x x}+I\right) P_{N} u\right\|_{L_{t}^{1} L_{x}^{2}} .
\end{aligned}
$$

On the other hand, a direct computation yields

$$
\left|\mathcal{F}_{t}^{-1}\left(\frac{\varphi_{N}(\xi)\left(\xi^{2}+1\right)}{i\left(\tau-\xi^{3}\right)+\xi^{2}+1}\right)\right|=C \varphi_{N}(\xi)\left(1+\xi^{2}\right) e^{-t\left(1+\xi^{2}\right)} \chi_{\mathbb{R}^{+}}(t)
$$

so that

$$
\left\|\mathcal{F}_{t}^{-1}\left(\frac{\varphi_{N}(\xi)\left(\xi^{2}+1\right)}{i\left(\tau-\xi^{3}\right)+\xi^{2}+1}\right)\right\|_{L_{t}^{1} L_{\xi}^{\infty}} \lesssim\left\|\langle N\rangle^{2} e^{-t\langle N\rangle^{2}} \chi_{\mathbb{R}^{+}}(t)\right\|_{L_{t}^{1}} \lesssim 1
$$

and the claim follows.

2. We show that for any fixed dyadic $N$, we have

$$
\left\|P_{N} u\right\|_{L^{2}} \lesssim\left\|P_{N} u\right\|_{\mathcal{S}^{-1}}
$$

Estimate (3.6) then follows after square-summing. Observe that (3.8) follows immediately from the estimate $\langle N\rangle^{-1}\left\langle L+N^{2}\right\rangle^{1 / 2} \gtrsim 1$ if the right-hand side is replaced by $\left\|P_{N} u\right\|_{X^{-1, \frac{1}{2}, 1}}$, so it suffices to prove (3.8) with $\left\|P_{N} u\right\|_{Y^{-1, \frac{1}{2}}}$ in the right-hand side. But applying again the Young and Hölder inequalities, this is easily verified:

$$
\begin{aligned}
\left\|P_{N} u\right\|_{L^{2}} & =\left\|\mathcal{F}_{t}^{-1}\left(\frac{1}{i\left(\tau-\xi^{3}\right)+\xi^{2}+1}\left(i\left(\tau-\xi^{3}\right)+\xi^{2}+1\right) \varphi_{N} \tilde{u}\right)\right\|_{L_{t \xi}^{2}} \\
& \lesssim\left\|\mathcal{F}_{t}^{-1}\left(\frac{\varphi_{N}(\xi)}{i\left(\tau-\xi^{3}\right)+\xi^{2}+1}\right)\right\|_{L_{t}^{2} L_{x}^{\infty}}\left\|P_{N} u\right\|_{Y^{0, \frac{1}{2}}} \\
& \lesssim\left\|e^{-t\langle N\rangle^{2}} \chi_{\mathbb{R}^{+}}(t)\right\|_{L_{t}^{2}}\left\|P_{N} u\right\|_{Y^{0, \frac{1}{2}}} \\
& \lesssim\langle N\rangle^{-1}\left\|P_{N} u\right\|_{Y^{0, \frac{1}{2}}} \lesssim\left\|P_{N} u\right\|_{Y^{-1, \frac{1}{2}}} .
\end{aligned}
$$

3. First it is clear from definitions that $\left(\sum_{L}\left[L^{1 / 2}\left\|Q_{L} u\right\|_{L^{2}}\right]^{2}\right)^{1 / 2} \lesssim\|u\|_{X^{0, \frac{1}{2}, 1}}$.

Setting now $v=\left(\partial_{t}+\partial_{x x x}\right) u$, we see that $u$ can be rewritten as

$$
u(t)=e^{-t \partial_{x x x}} u(0)+\int_{0}^{t} e^{-\left(t-t^{\prime}\right) \partial_{x x x}} v\left(t^{\prime}\right) d t^{\prime} .
$$


By virtue of Lemma 3.1, we have

$$
\left(\sum_{L}\left[L^{1 / 2}\left\|Q_{L} e^{-t \partial_{x x x}} u(0)\right\|_{L^{2}}\right]^{2}\right)^{1 / 2} \lesssim\|u(0)\|_{L^{2}} \lesssim\|u\|_{L_{t}^{\infty} L_{x}^{2}} .
$$

Moreover, we get as previously

$$
\|u\|_{L_{t}^{\infty} L_{x}^{2}} \lesssim\left\|\mathcal{F}_{t}^{-1}\left(\frac{1}{i\left(\tau-\xi^{3}\right)+\xi^{2}+1}\right)\right\|_{L_{t \xi}^{\infty}}\|u\|_{Y^{0, \frac{1}{2}}} \lesssim\|u\|_{Y^{0, \frac{1}{2}}} .
$$

Thanks to estimate (3.5), it remains to show that

$$
\left(\sum_{L}\left[L^{1 / 2}\left\|Q_{L} \int_{0}^{t} e^{-\left(t-t^{\prime}\right) \partial_{x x x}} v\left(t^{\prime}\right) d t^{\prime}\right\|_{L^{2}}\right]^{2}\right)^{1 / 2} \lesssim\|v\|_{L_{t}^{1} L_{x}^{2}}
$$

In order to prove this, we split the integral $\int_{0}^{t}=\int_{-\infty}^{t}-\int_{-\infty}^{0}$. By Lemma 3.1, the contribution of the first term is bounded by

$$
\lesssim\left\|\int_{-\infty}^{0} e^{t^{\prime} \partial_{x x x}} v\left(t^{\prime}\right) d t^{\prime}\right\|_{L_{x}^{2}} \lesssim\|v\|_{L_{t}^{1} L_{x}^{2}}
$$

For the last term, by Minkowski it suffices to show that

$$
\left(\sum_{L}\left[L^{1 / 2}\left\|Q_{L}\left(\chi_{t>t^{\prime}} e^{-\left(t-t^{\prime}\right) \partial_{x x x}} v\left(t^{\prime}\right)\right)\right\|_{L_{t x}^{2}}\right]^{2}\right)^{1 / 2} \lesssim\left\|v\left(t^{\prime}\right)\right\|_{L_{x}^{2}} .
$$

This can be proved by a time-restriction argument. Indeed, for any $T>0$, we have

$$
\begin{aligned}
& \left(\sum_{L}\left[L^{1 / 2}\left\|Q_{L}\left(\eta_{T}(t) \chi_{t>t^{\prime}} e^{-\left(t-t^{\prime}\right) \partial_{x x x}} v\left(t^{\prime}\right)\right)\right\|_{L^{2}}\right]^{2}\right)^{1 / 2} \\
& \quad \lesssim\left\||\tau|^{1 / 2} \widehat{v}\left(t^{\prime}\right) \mathcal{F}_{t}\left(\eta_{T}(t) \chi_{t>t^{\prime}}\right)(\tau)\right\|_{L^{2}} \\
& \quad \lesssim\left\|v\left(t^{\prime}\right)\right\|_{L^{2}}\left\||\tau|^{1 / 2} \mathcal{F}_{t}\left(\eta(t) \chi_{t T>t^{\prime}}\right)\right\|_{L^{2}} \\
& \quad \lesssim\left\|v\left(t^{\prime}\right)\right\|_{L^{2}}
\end{aligned}
$$

We conclude by passing to the limit $T \rightarrow \infty$.

We now state a general and classical result which ensures that our resolution space is well compatible with the dispersive properties of the Airy equation. Actually, it is a direct consequence of Lemma 4.1 in [19] together with the fact that the resolution space $\mathcal{S}_{0}$ used by Tao to solve $4-\mathrm{KdV}$ contains our space $\mathcal{S}^{0}$ thanks to estimate (3.5) 
Lemma 3.3 (Extension lemma). Let $Z$ be a Banach space of functions on $\mathbb{R} \times \mathbb{R}$ with the property that

$$
\|g(t) u(t, x)\|_{Z} \lesssim\|g\|_{L_{t}^{\infty}}\|u(t, x)\|_{Z}
$$

holds for any $u \in Z$ and $g \in L_{t}^{\infty}(\mathbb{R})$. Let $T$ be a spacial linear operator for which one has the estimate

$$
\left\|T\left(e^{-t \partial_{x x x}} P_{N} \phi\right)\right\|_{Z} \lesssim\left\|P_{N} \phi\right\|_{L^{2}}
$$

for some dyadic $N$ and for all $\phi$. Then one has the embedding

$$
\left\|T\left(P_{N} u\right)\right\|_{Z} \lesssim\left\|P_{N} u\right\|_{\mathcal{S}^{0}} .
$$

Combined with the unitarity of the Airy group in $L^{2}$ and the sharp Kato smoothing effect

$$
\left\|\partial_{x} e^{-t \partial_{x x x}} \phi\right\|_{L_{x}^{\infty} L_{t}^{2}} \lesssim\|\phi\|_{L^{2}}, \quad \forall \phi \in L^{2},
$$

we deduce the following result.

Corollary 3.4. For any $u$, we have $e^{2}$

$$
\begin{gathered}
\|u\|_{L_{t}^{\infty} H_{x}^{-1}} \lesssim\|u\|_{\mathcal{S}^{-1}}, \\
\left\|P_{N} u\right\|_{L_{x}^{\infty} L_{t}^{2}} \lesssim N^{-1}\left\|P_{N} u\right\|_{\mathcal{S}^{0}},
\end{gathered}
$$

provided the right-hand side is finite. In particular, $\mathcal{S}^{-1} \hookrightarrow L_{t}^{\infty} H^{-1}$.

\section{Linear estimates}

In this section we prove linear estimates related to the operator $W$ as well as to the extension of the Duhamel operator introduced in (3.4).

At this this stage let us give some explanations on our choice of this extension. Let us keep in mind that this extension has to be compatible with linear estimates in both norms $X^{s, 1 / 2,1}$ and $Y^{s, 1 / 2}$. First, since $X^{s, 1 / 2,1}$ is a Besov in time space we are not allowed to simply multiply the Duhamel term by $\chi_{\mathbb{R}^{+}}(t)$. Second, in order to prove the desired linear estimate in $Y^{s, 1 / 2}$ the strategy is to use the fact that the Duhamel term satisfies a forced KdV-B equation. Unfortunately, it turns out that the extension introduced in [16], that makes the calculus simple, does not satisfy such a PDE for negative time. The new extension that we introduce in this work has the properties to satisfy some forced PDE related to KdV-B for negative times (see (4.14)) and to be compatible with linear estimates in $X^{s, 1 / 2,1}$. However the proof is now a little more complicated even if it follows the same lines as that of [16, Propositions 2.3], see also [11, Proposition 4.4].

The following lemma is a dyadic version of [16, Proposition 2.1].

${ }^{2}$ Note that (3.12) can also be deduced from the estimate (3.9). 
Proposition 4.1. For all $\phi \in H^{-1}(\mathbb{R})$, we have

$$
\|\eta(t) W(t) \phi\|_{\mathcal{S}^{-1}} \lesssim\|\phi\|_{H^{-1}}
$$

Proof. We are going to bound the left-hand side in (4.1) by the $X^{-1, \frac{1}{2}, 1}$-norm of $\eta(t) W(t) \phi$. This is equivalent to prove that

$$
\sum_{L}\left\langle L+N^{2}\right\rangle^{1 / 2}\left\|P_{N} Q_{L}(\eta(t) W(t) \phi)\right\|_{L_{x t}^{2}} \lesssim\left\|P_{N} \phi\right\|_{L^{2}}
$$

for each dyadic $N$. Using Plancherel, we obtain

$$
\begin{aligned}
& \sum_{L}\left\langle L+N^{2}\right\rangle^{1 / 2}\left\|P_{N} Q_{L}(\eta(t) W(t) \phi)\right\|_{L_{x t}^{2}} \\
& \quad \lesssim \sum_{L}\left\langle L+N^{2}\right\rangle^{1 / 2}\left\|\varphi_{N}(\xi) \varphi_{L}(\tau) \widehat{\phi}(\xi) \mathcal{F}_{t}\left(\eta(t) e^{-|t| \xi^{2}}\right)(\tau)\right\|_{L_{\tau \xi}^{2}} \\
& \quad \lesssim\left\|P_{N} \phi\right\|_{L^{2}} \sum_{L}\left\langle L+N^{2}\right\rangle^{1 / 2}\left\|\varphi_{N}(\xi) P_{L}\left(\eta(t) e^{-|t| \xi^{2}}\right)\right\|_{L_{\xi}^{\infty} L_{t}^{2}}
\end{aligned}
$$

Hence it remains to show that

$$
\sum_{L}\left\langle L+N^{2}\right\rangle^{1 / 2}\left\|\varphi_{N}(\xi) P_{L}\left(\eta(t) e^{-|t| \xi^{2}}\right)\right\|_{L_{\xi}^{\infty} L_{t}^{2}} \lesssim 1 .
$$

We split the summand into $L \leq\langle N\rangle^{2}$ and $L \geq\langle N\rangle^{2}$. In the former case, we get by Bernstein

$$
\begin{aligned}
\sum_{L \leq\langle N\rangle^{2}}\left\langle L+N^{2}\right\rangle^{1 / 2}\left\|\varphi_{N}(\xi) P_{L}\left(\eta(t) e^{-|t| \xi^{2}}\right)\right\|_{L_{\xi}^{\infty} L_{t}^{2}} & \\
& \lesssim \sum_{L \leq\langle N\rangle^{2}}\langle N\rangle L^{1 / 2} \sup _{|\xi| \sim N}\left\|\eta(t) e^{-|t| \xi^{2}}\right\|_{L_{t}^{1}}
\end{aligned}
$$

Noticing that one can bound $\left\|\eta(t) e^{-|t| \xi^{2}}\right\|_{L^{1}}$ either by $\|\eta\|_{L^{1}}$ or by $\left\|e^{-|t| \xi^{2}}\right\|_{L_{t}^{1}} \sim$ $|\xi|^{-2}$, we infer that

$$
\sum_{L \leq\langle N\rangle^{2}}\left\langle L+N^{2}\right\rangle^{1 / 2}\left\|\varphi_{N}(\xi) P_{L}\left(\eta(t) e^{-|t| \xi^{2}}\right)\right\|_{L_{\xi}^{\infty} L_{t}^{2}} \lesssim\langle N\rangle^{2} \min \left(1, N^{-2}\right) \lesssim 1
$$

Now we deal with the case $L \geq\langle N\rangle^{2}$. A standard paraproduct rearrangement allows us to write

$$
\begin{aligned}
P_{L}\left(\eta(t) e^{-|t| \xi^{2}}\right) & =P_{L}\left(\sum_{M \gtrsim L}\left(P_{M} \eta(t) P_{\lesssim M} e^{-|t| \xi^{2}}+P_{\lesssim M} \eta(t) P_{M} e^{-|t| \xi^{2}}\right)\right. \\
& =P_{L}(I)+P_{L}(I I) .
\end{aligned}
$$


Using Schur's test, the term $P_{L}(I)$ is directly bounded by

$$
\begin{aligned}
& \sum_{L \geq\langle N\rangle^{2}}\left\langle L+N^{2}\right\rangle^{1 / 2}\left\|\varphi_{N} P_{L}(I)\right\|_{L_{\xi}^{\infty} L_{t}^{2}} \\
& \quad \lesssim \sum_{L} L^{1 / 2} \sum_{M \gtrsim L}\left\|\varphi_{N} P_{M} \eta(t)\right\|_{L_{\xi}^{\infty} L_{t}^{2}}\left\|\varphi_{N} P_{\lesssim M} e^{-|t| \xi^{2}}\right\|_{L_{\xi t}^{\infty}} \\
& \quad \lesssim \sum_{M} M^{1 / 2}\left\|P_{M} \eta\right\|_{L_{t}^{2}} \lesssim 1 .
\end{aligned}
$$

Similarly for $P_{L}(I I)$, we have

$$
\begin{aligned}
& \sum_{L \geq\langle N\rangle^{2}}\left\langle L+N^{2}\right\rangle^{1 / 2}\left\|\varphi_{N} P_{L}(I I)\right\|_{L_{\xi}^{\infty} L_{t}^{2}} \\
& \quad \lesssim \sum_{L} L^{1 / 2} \sum_{M \gtrsim L}\left\|\varphi_{N} P_{\lesssim M} \eta(t)\right\|_{L_{\xi t}^{\infty}}\left\|\varphi_{N} P_{M} e^{-|t| \xi^{2}}\right\|_{L_{\xi}^{\infty} L_{t}^{2}} \\
& \quad \lesssim \sum_{M} M^{1 / 2}\left\|\varphi_{N} P_{M} e^{-|t| \xi^{2}}\right\|_{L_{\xi}^{\infty} L_{t}^{2}}
\end{aligned}
$$

Moreover, it is not too hard to check that if $|\xi| \sim N$, then $\left\|P_{M} e^{-|t| \xi^{2}}\right\|_{L_{t}^{2}} \lesssim$ $\left\|P_{M} e^{-|t| N^{2}}\right\|_{L_{t}^{2}}$, thus

$$
\sum_{L \geq\langle N\rangle^{2}}\left\langle L+N^{2}\right\rangle^{1 / 2}\left\|\varphi_{N} P_{L}(I I)\right\|_{L_{\xi}^{\infty} L_{t}^{2}} \lesssim \sum_{M} M^{1 / 2}\left\|P_{M} e^{-|t| N^{2}}\right\|_{L_{t}^{2}} \lesssim 1
$$

where we used the fact that the Besov space $\dot{B}_{2,1}^{1 / 2}$ has a scaling invariance and $e^{-|t|} \in \dot{B}_{2,1}^{1 / 2}$.

Lemma 4.2. For $w \in \mathcal{S}\left(\mathbb{R}^{2}\right)$, consider $k_{\xi}$ defined on $\mathbb{R}$ by

$$
k_{\xi}(t)=\eta(t) \varphi_{N}(\xi) \int_{\mathbb{R}} \frac{e^{i t \tau} e^{(t-|t|) \xi^{2}}-e^{-|t| \xi^{2}}}{i \tau+\xi^{2}} \widetilde{w}(\tau) d \tau
$$

Then, for all $\xi \in \mathbb{R}$, it holds

$$
\sum_{L}\left\langle L+N^{2}\right\rangle^{1 / 2}\left\|P_{L} k_{\xi}\right\|_{L_{t}^{2}} \lesssim \sum_{L}\left\langle L+N^{2}\right\rangle^{-1 / 2}\left\|\varphi_{L}(\tau) \varphi_{N}(\xi) \widetilde{w}\right\|_{L_{\tau}^{2}} .
$$


Proof. Following [16], we rewrite $k_{\xi}$ as

$$
\begin{aligned}
k_{\xi}(t)= & \eta(t) e^{(t-|t|) \xi^{2}} \int_{|\tau| \leq 1} \frac{e^{i t \tau}-1}{i \tau+\xi^{2}} \widetilde{w_{N}}(\tau) d \tau \\
& +\eta(t) \int_{|\tau| \leq 1} \frac{e^{(t-|t|) \xi^{2}-e^{-|t| \xi^{2}}}}{i \tau+\xi^{2}} \widetilde{w_{N}}(\tau) d \tau \\
& +\eta(t) e^{(t-|t|) \xi^{2}} \int_{|\tau| \geq 1} \frac{e^{i t \tau}}{i \tau+\xi^{2}} \widetilde{w_{N}}(\tau) d \tau-\eta(t) \int_{|\tau| \geq 1} \frac{e^{-|t| \xi^{2}}}{i \tau+\xi^{2}} \widetilde{w_{N}}(\tau) d \tau \\
= & I+I I+I I I-I V
\end{aligned}
$$

where $w_{N}$ is defined by $\mathcal{F}_{x}\left(w_{N}\right)(\xi)=\varphi_{N}(\xi) \mathcal{F}_{x}(w)(\xi)$.

Contribution of $I V$. Clearly we have

$$
\left\|P_{L}(I V)\right\|_{L_{t}^{2}} \lesssim\left\|P_{L}\left(\eta(t) e^{-|t| \xi^{2}}\right)\right\|_{L_{t}^{2}} \int_{|\tau| \geq 1} \frac{\left|\widetilde{w_{N}}(\tau)\right|}{\left\langle i \tau+\xi^{2}\right\rangle} d \tau
$$

On the other hand, by Cauchy-Schwarz in $\tau$,

$$
\int_{|\tau| \geq 1} \frac{\left|\widetilde{w_{N}}(\tau)\right|}{\left\langle i \tau+\xi^{2}\right\rangle} d \tau \lesssim \sum_{L}\left\langle L+N^{2}\right\rangle^{-1}\left\|\varphi_{L} \widetilde{w_{N}}\right\|_{L_{\tau}^{1}} \lesssim \sum_{L}\left\langle L+N^{2}\right\rangle^{-1 / 2}\left\|\varphi_{L} \widetilde{w_{N}}\right\|_{L_{\tau}^{2}},
$$

which combined with (4.3) yields the desired bound.

Contribution of $I I$. By the Cauchy-Schwarz inequality,

$$
\begin{aligned}
\left\|P_{L}(I I)\right\|_{L_{t}^{2}} \lesssim & \left\|P_{L}\left(\eta(t)\left(e^{(t-|t|) \xi^{2}}-e^{-\left.|t| \xi\right|^{2}}\right)\right)\right\|_{L_{t}^{2}} \\
& \times\left(\int \frac{\left|\widetilde{w_{N}}(\tau)\right|^{2}}{\left\langle i \tau+\xi^{2}\right\rangle} d \tau\right)^{1 / 2}\left(\int_{|\tau| \leq 1} \frac{\left\langle i \tau+\xi^{2}\right\rangle}{\left|i \tau+\xi^{2}\right|^{2}} d \tau\right)^{1 / 2} \\
\lesssim & \left\|P_{L}\left(\eta(t)\left(e^{(t-|t|) \xi^{2}}-e^{-\left.|t| \xi\right|^{2}}\right)\right)\right\|_{L_{t}^{2}} \\
& \times N^{-2}\langle N\rangle \sum_{L}\left\langle L+N^{2}\right\rangle^{-1 / 2}\left\|\varphi_{L} \widetilde{w_{N}}\right\|_{L_{\tau}^{2}}
\end{aligned}
$$

Hence we need to estimate

$$
\begin{aligned}
\sum_{L}\langle L+ & \left.N^{2}\right\rangle^{1 / 2}\left\|P_{L}\left(\eta(t)\left(e^{(t-|t|) \xi^{2}}-e^{-\left.|t| \xi\right|^{2}}\right)\right)\right\|_{L_{t}^{2}} \\
& \lesssim \sum_{L}\left\langle L+N^{2}\right\rangle^{1 / 2}\left(\left\|P_{L}\left(\eta(t) e^{(t-|t|) \xi^{2}}\right)\right\|_{L_{t}^{2}}+\left\|P_{L}\left(\eta(t) e^{-|t| \xi^{2}}\right)\right\|_{L_{t}^{2}}\right) .
\end{aligned}
$$


The second term in the right-hand side is bounded by 1 thanks to estimate(4.3). Setting $\theta(t)=\eta(t) e^{(t-|t|) \xi^{2}}$, it is not too hard to check that one integration by parts yields $|\hat{\theta}(\tau)| \lesssim \frac{1}{|\tau|}$ whereas two integrations yield $|\hat{\theta}(\tau)| \lesssim \frac{\langle\xi\rangle^{2}}{|\tau|^{2}}$. We thus infer that

$$
\begin{aligned}
& \sum_{L}\left\langle L+N^{2}\right\rangle^{1 / 2}\left\|\varphi_{L} \hat{\theta}\right\|_{L_{\tau}^{2}} \lesssim \sum_{L \leq 1}\langle N\rangle L^{1 / 2}\|\theta\|_{L_{t}^{1}} \\
& +\sum_{1 \leq L \leq\langle N\rangle^{2}} \frac{\langle N\rangle}{L^{1 / 2}}+\sum_{L \geq\langle N\rangle^{2}}\langle L\rangle^{1 / 2} \frac{\langle N\rangle^{2}}{L^{3 / 2}} \lesssim\langle N\rangle .
\end{aligned}
$$

This provides the result for $N \geq 1$. In the case $N \leq 1$, we use a Taylor expansion and obtain

$$
\begin{aligned}
\| P_{L}\left(\eta(t)\left(e^{(t-|t|) \xi^{2}}-1+1-e^{-|t| \xi^{2}}\right) \|_{L_{t}^{2}}\right. & \\
& \lesssim \sum_{n \geq 1} \frac{|\xi|^{2 n}}{n !}\left(\left\|P_{L}\left(|t|^{n} \eta(t)\right)\right\|_{L_{t}^{2}}+2^{n}\left\|P_{L}\left(t^{n} \eta(t) \chi \mathbb{R}_{-}(t)\right)\right\|_{L_{t}^{2}}\right) .
\end{aligned}
$$

According to the Sobolev embedding $H^{1} \hookrightarrow B_{2,1}^{1 / 2}$ as well as the estimate $\left\|\chi \mathbb{R}_{-} f\right\|_{H^{1}} \lesssim$ $\|f\|_{H^{1}}$ provided $f(0)=0$, we deduce

$$
\begin{aligned}
& \sum_{L}\left\langle L+N^{2}\right\rangle^{1 / 2}\left\|P_{L}\left(\eta(t)\left(e^{(t-|t|) \xi^{2}}-e^{-|t| \xi^{2}}\right)\right)\right\|_{L_{t}^{2}} \\
& \quad \lesssim \xi^{2} \sum_{n \geq 1} \frac{1}{n !}\left(\left\||t|^{n} \eta(t)\right\|_{B_{2,1}^{1 / 2}}+2^{n}\left\|t^{n} \eta(t) \chi \mathbb{R}_{-}(t)\right\|_{\left.B_{2,1}^{1 / 2}\right)}\right. \\
& \quad \lesssim N^{2} \sum_{n \geq 1} \frac{2^{n}}{n !}\left\||t|^{n} \eta(t)\right\|_{H_{t}^{1}} \lesssim N^{2} .
\end{aligned}
$$

Gathering this and (4.4) we conclude that

$$
\sum_{L}\left\langle L+N^{2}\right\rangle^{1 / 2}\left\|P_{L}(I I)\right\|_{L_{t}^{2}} \lesssim \sum_{L}\left\langle L+N^{2}\right\rangle^{-1 / 2}\left\|\varphi_{L} \widetilde{w_{N}}\right\|_{L_{\tau}^{2}} .
$$

Contribution of $I$. Since $I$ can be rewritten as

$$
I=\eta(t) e^{(t-|t|) \xi^{2}} \int_{|\tau| \leq 1} \sum_{n \geq 1} \frac{(i t \tau)^{n}}{n !} \frac{\widetilde{w_{N}}(\tau)}{i \tau+\xi^{2}} d \tau,
$$

we have

$$
\left\|P_{L}(I)\right\|_{L_{t}^{2}} \lesssim \sum_{n \geq 1} \frac{1}{n !}\left\|P_{L}\left(t^{n} \theta(t)\right)\right\|_{L_{t}^{2}} \int_{|\tau| \leq 1} \frac{|\tau|^{n}}{\left|i \tau+\xi^{2}\right|}\left|\widetilde{w_{N}}(\tau)\right| d \tau .
$$


Using Cauchy-Schwarz we get, for $n \geq 1$,

$$
\begin{aligned}
\int_{|\tau| \leq 1} \frac{|\tau|^{n}}{\left|i \tau+\xi^{2}\right|} \mid \widetilde{w_{N}}(\tau) d \tau & \lesssim\left(\int \frac{\left|\widetilde{w_{N}}(\tau)\right|^{2}}{\left\langle i \tau+\xi^{2}\right\rangle} d \tau\right)^{1 / 2}\left(\int_{|\tau| \leq 1} \frac{|\tau|^{2}\left\langle i \tau+\xi^{2}\right\rangle}{\left|i \tau+\xi^{2}\right|^{2}} d \tau\right)^{1 / 2} \\
& \lesssim\langle N\rangle^{-1} \sum_{L}\left\langle L+N^{2}\right\rangle^{-1 / 2}\left\|\varphi_{L} \widetilde{w_{N}}\right\|_{L_{\tau}^{2}} .
\end{aligned}
$$

Therefore, it suffices to show that (see above the contribution of $I I$ for the definition of $\theta$ )

$$
\sum_{L}\left\langle L+N^{2}\right\rangle^{1 / 2} \sum_{n \geq 1} \frac{1}{n !}\left\|P_{L}\left(t^{n} \theta(t)\right)\right\|_{L_{t}^{2}} \lesssim\langle N\rangle
$$

But again we have $\left|\mathcal{F}_{t}\left(t^{n} \theta(t)\right)\right| \lesssim 2^{n} \min \left(\frac{1}{|\tau|}, \frac{\langle\xi\rangle^{2}}{\tau^{2}}\right)$ and arguing as in (4.5), we get

$$
\sum_{L}\left\langle L+N^{2}\right\rangle^{1 / 2} \sum_{n \geq 1} \frac{1}{n !}\left\|P_{L}\left(t^{n} \theta(t)\right)\right\|_{L_{t}^{2}} \lesssim \sum_{n \geq 1}\langle N\rangle \frac{2^{n}}{n !} \lesssim\langle N\rangle .
$$

Contribution of $I I I$. Setting $\hat{g}(\tau)=\frac{\widetilde{w_{N}}(\tau)}{i \tau+\xi^{2}} \chi_{|\tau| \geq 1}$, we have to prove

$$
\sum_{L}\left\langle L+N^{2}\right\rangle^{1 / 2}\left\|P_{L}(\theta g)\right\|_{L_{t}^{2}} \lesssim \sum_{L}\left\langle L+N^{2}\right\rangle^{1 / 2}\left\|P_{L} g\right\|_{L_{t}^{2}}
$$

Using the paraproduct decomposition, we have

$$
P_{L}(\theta g)=P_{L}\left(\sum_{M \gtrsim L}\left(P_{\lesssim M} \theta P_{\sim M} g+P_{\sim M} \theta P_{\lesssim M} g\right)\right)=P_{L}\left(I I I_{1}\right)+P_{L}\left(I I I_{2}\right)
$$

and we estimate the contributions of these two terms separately.

Contribution of $I I I_{1}$. The sum over $L \geq\langle N\rangle^{2}$ is estimated in the following way:

$$
\begin{aligned}
\sum_{L \geq\langle N\rangle^{2}}\left\langle L+N^{2}\right\rangle^{1 / 2}\left\|P_{L}\left(I I I_{1}\right)\right\|_{L_{t}^{2}} & \lesssim \sum_{L \geq\langle N\rangle^{2}}\langle L\rangle^{1 / 2} \sum_{M \gtrsim L}\left\|P_{\lesssim M} \theta\right\|_{L_{t}^{\infty}}\left\|P_{M} g\right\|_{L_{t}^{2}} \\
& \lesssim \sum_{M}\langle M\rangle^{1 / 2}\left\|P_{M} g\right\|_{L_{t}^{2} .}
\end{aligned}
$$

Now we deal with the case where $L \lesssim\langle N\rangle^{2}$. If $\hat{\theta}$ is localized in an annulus $\{|\tau| \sim$ 
$M$ \}, we get from the Bernstein inequality that

$$
\begin{aligned}
\sum_{L \leq\langle N\rangle^{2}}\left\langle L+N^{2}\right\rangle^{1 / 2} \sum_{M \gtrsim L}\left\|P_{L}\left(P_{M} \theta P_{M} g\right)\right\|_{L_{t}^{2}} \\
\quad \lesssim \sum_{M}\langle N\rangle \sum_{L \lesssim M} L^{1 / 2}\left\|P_{M} \theta P_{M} g\right\|_{L_{t}^{1}} \\
\lesssim \sum_{M}\langle N\rangle M^{1 / 2}\left\|P_{M} \theta\right\|_{L_{t}^{2}}\left\|P_{M} g\right\|_{L^{2}} \\
\lesssim \sum_{M}\langle N\rangle\left\|P_{M} g\right\|_{L_{t}^{2}},
\end{aligned}
$$

where we used the estimate $\left\|P_{M} \theta\right\|_{L_{t}^{2}} \lesssim\left\|\frac{\varphi_{M}(\tau)}{\tau}\right\|_{L_{\tau}^{2}} \lesssim M^{-1 / 2}$. If $\hat{\theta}$ is localized in a ball $\{|\tau| \ll M\}$, then we must have $M \sim L$ and thus

$$
\sum_{L \leq\langle N\rangle^{2}}\left\langle L+N^{2}\right\rangle^{1 / 2} \sum_{M \sim L}\left\|P_{L}\left(P_{\ll M} \theta P_{M} g\right)\right\|_{L_{t}^{2}} \lesssim \sum_{L}\langle N\rangle\left\|P_{\ll L} \theta\right\|_{L_{t}^{\infty}}\left\|P_{L} g\right\|_{L_{t}^{2}},
$$

which is acceptable.

Contribution of $I I I_{2}$. Consider the case $L \geq\langle N\rangle^{2}$. Since $|\hat{\theta}| \lesssim \frac{\langle\xi\rangle^{2}}{\tau^{2}}$, we have

$$
\left\|P_{L}\left(P_{M} \theta P_{\lesssim M} g\right)\right\|_{L_{t}^{2}} \lesssim\left\|\varphi_{M} \hat{\theta}\right\|_{L_{\tau}^{1}}\left\|P_{\lesssim M} g\right\|_{L_{t}^{2}} \lesssim \frac{\langle N\rangle^{2}}{M}\|g\|_{L_{t}^{2}} .
$$

It follows that

$$
\sum_{L \geq\left\langle N^{2}\right\rangle}\left\langle L+N^{2}\right\rangle^{1 / 2}\left\|P_{L}\left(I I I_{2}\right)\right\|_{L_{t}^{2}} \lesssim \sum_{M \gtrsim\langle N\rangle^{2}} M^{1 / 2} \frac{\langle N\rangle^{2}}{M}\|g\|_{L_{t}^{2}} \lesssim\langle N\rangle\|g\|_{L_{t}^{2}} .
$$

It remains to establish the bound in the case $L \leq\langle N\rangle^{2}$. We may assume that $\hat{g}$ is supported in a ball $\{|\tau| \ll M\}$ since the other case was already treated (cf. estimate (4.7)). Therefore, $M \sim L$ and

$$
\begin{aligned}
\sum_{L \leq\langle N\rangle^{2}}\left\langle L+N^{2}\right\rangle^{1 / 2} \sum_{M \gtrsim L}\left\|P_{L}\left(P_{M} \theta P_{\ll M} g\right)\right\|_{L_{t}^{2}} \\
\quad \lesssim \sum_{L}\langle N\rangle\left\|P_{L} \theta P_{\ll L L} g\right\|_{L_{t}^{2}} \\
\quad \lesssim \sum_{L}\langle N\rangle\left\|P_{L} \theta\right\|_{L_{t}^{2}} \sum_{M \ll L}\left\|P_{M} g\right\|_{L_{t}^{\infty}} \\
\lesssim \sum_{L}\langle N\rangle L^{-1 / 2} \sum_{M \ll L} M^{1 / 2}\left\|P_{M} g\right\|_{L_{t}^{2}} \\
\lesssim \sum_{M}\langle N\rangle\left\|P_{M} g\right\|_{L_{t}^{2}} .
\end{aligned}
$$

This completes the proof of Lemma 4.2. 
Proposition 4.3. Let $\mathcal{L}: f \mapsto \mathcal{L} f$ denote the linear operator

$$
\begin{aligned}
\mathcal{L} f(t, x)= & \eta(t)\left(\chi_{\mathbb{R}^{+}}(t) \int_{0}^{t} W\left(t-t^{\prime}, t-t^{\prime}\right) f\left(t^{\prime}\right) d t^{\prime}\right. \\
& \left.+\chi_{\mathbb{R}^{-}}(t) \int_{0}^{t} W\left(t-t^{\prime}, t+t^{\prime}\right) f\left(t^{\prime}\right) d t^{\prime}\right) .
\end{aligned}
$$

If $f \in \mathcal{N}^{-1}$, then

$$
\|\mathcal{L} f\|_{\mathcal{S}^{-1}} \lesssim\|f\|_{\mathcal{N}^{-1}}
$$

Proof. It suffices to show that

$$
\|\mathcal{L} f\|_{X^{-1, \frac{1}{2}, 1}} \lesssim\|f\|_{X^{-1,-\frac{1}{2},-1}}
$$

and

$$
\|\mathcal{L} f\|_{Y^{-1, \frac{1}{2}}} \lesssim\|f\|_{Y^{-1,-\frac{1}{2}}}
$$

Taking the $x$-Fourier transform, we get

$$
\begin{aligned}
\mathcal{L} f(t, x)= & U(t)\left[\chi \chi_{\mathbb{R}+}(t) \eta(t) \int_{\mathbb{R}} e^{i x \xi} \int_{0}^{t} e^{-\left|t-t^{\prime}\right| \xi^{2}} \mathcal{F}_{x}\left(U\left(-t^{\prime}\right) f\left(t^{\prime}\right)\right)(\xi) d t^{\prime} d \xi\right. \\
& \left.+\chi_{\mathbb{R}-}(t) \eta(t) \int_{\mathbb{R}} e^{i x \xi} \int_{0}^{t} e^{-\left|t+t^{\prime}\right| \xi^{2}} \mathcal{F}_{x}\left(U\left(-t^{\prime}\right) f\left(t^{\prime}\right)\right)(\xi) d t^{\prime} d \xi\right] \\
= & U(t)\left[\eta(t) \int_{\mathbb{R}} e^{i x \xi} \int_{0}^{t} e^{-|t| \xi^{2}} e^{t^{\prime} \xi^{2}} \mathcal{F}_{x}\left(U\left(-t^{\prime}\right) f\left(t^{\prime}\right)\right)(\xi) d t^{\prime} d \xi\right] .
\end{aligned}
$$

Setting $w\left(t^{\prime}\right)=U\left(-t^{\prime}\right) f\left(t^{\prime}\right)$, and using the time Fourier transform, we infer that

$$
\mathcal{L} f(t, x)=U(t)\left[\eta(t) \int_{\mathbb{R}^{2}} e^{i x \xi} \frac{e^{i t \tau} e^{(t-|t|) \xi^{2}}-e^{-|t| \xi^{2}}}{i \tau+\xi^{2}} \tilde{w}(\tau, \xi) d \tau d \xi\right] .
$$

Estimate (4.10) follows then easily from Lemma 4.2.

Now we turn to estimate (4.11). It is equivalent to prove that for any dyadic $N$,

$$
\left\|\left(\partial_{t}+\partial_{x x x}-\partial_{x x}+I\right) P_{N} \mathcal{L} f\right\|_{L_{t}^{1} L_{x}^{2}} \lesssim\left\|P_{N} f\right\|_{L_{t}^{1} L_{x}^{2}}
$$

In view of the expression of $\mathcal{L}$ it suffices to prove (4.12) separately for $\chi_{\mathbb{R}}+\mathcal{L} f$ and $\chi_{\mathbb{R}^{-}} \mathcal{L} f$. First, a straightforward calculation leads to

$$
\begin{aligned}
\left(\partial_{t}+\right. & \left.\partial_{x x x}-\partial_{x x}+I\right)\left(\chi_{\mathbb{R}^{+}} \mathcal{L} f(t)\right) \\
& =\eta(t) \chi_{\mathbb{R}^{+}}(t) f(t)+\left(\eta^{\prime}(t)+\eta(t)\right) \chi_{\mathbb{R}^{+}}(t) \int_{0}^{t} W\left(t-t^{\prime}, t-t^{\prime}\right) f\left(t^{\prime}\right) d t^{\prime}
\end{aligned}
$$


Computing the $L_{t}^{1} L_{x}^{2}$ norm, we get

$$
\begin{aligned}
\|\left(\partial_{t}+\partial_{x x x}\right. & \left.-\partial_{x x}+I\right) P_{N}\left(\chi_{\mathbb{R}^{+}} \mathcal{L} f\right) \|_{L_{t}^{1} L_{x}^{2}} \\
& \lesssim\|f\|_{L_{t}^{1} L_{x}^{2}}+\left\|\eta^{\prime}+\eta\right\|_{L_{t}^{1}} \sup _{t} \int_{0}^{\infty}\left\|e^{i\left(t-t^{\prime}\right) \xi^{3}} e^{-\left(t-t^{\prime}\right) \xi^{2}} \widehat{f}\left(t^{\prime}\right)\right\|_{L_{\xi}^{2}} d t^{\prime},
\end{aligned}
$$

and estimate (4.12) follows.

Now, let us tackle the proof for $\chi_{\mathbb{R}^{-}} \mathcal{L} f$. We have to work a little more since clearly $\mathcal{L} f$ does not satisfy the same equation for negative times. Actually, one can check that

$$
\begin{aligned}
& \left(\partial_{t}+\partial_{x x x}+\partial_{x x}+I\right)\left(\chi_{\mathbb{R}^{-}} \mathcal{L} f(t)\right) \\
= & \eta(t) \chi_{\mathbb{R}^{-}}(t) W(0,2 t) f(t)+\left(\eta^{\prime}(t)+\eta(t)\right) \chi_{\mathbb{R}^{-}}(t) \int_{0}^{t} W\left(t-t^{\prime}, t+t^{\prime}\right) f\left(t^{\prime}\right) d t^{\prime} .
\end{aligned}
$$

and thus

$$
\begin{aligned}
& \left(\partial_{t}+\partial_{x x x}-\partial_{x x}+I\right)\left(\chi_{\mathbb{R}^{-}} \mathcal{L} f(t)\right)=-2 \partial_{x x}\left(\chi_{\mathbb{R}^{-}} \mathcal{L} f(t)\right) \\
+ & \eta(t) \chi_{\mathbb{R}^{-}}(t) W(2 t, 0) f(t)+\left(\eta^{\prime}(t)+\eta(t)\right) \chi_{\mathbb{R}^{-}}(t) \int_{0}^{t} W\left(t-t^{\prime}, t+t^{\prime}\right) f\left(t^{\prime}\right) d t^{\prime} .
\end{aligned}
$$

Setting $w:=P_{N}\left(\chi_{\mathbb{R}^{-}} \mathcal{L} f(t)\right)$ and $g:=\eta(t) \chi_{\mathbb{R}^{-}}(t) W(2 t, 0) f(t)+\left(\eta^{\prime}(t)+\eta(t)\right) \chi_{\mathbb{R}^{-}}(t) \int_{0}^{t} W\left(t-t^{\prime}, t+t^{\prime}\right) f\left(t^{\prime}\right) d t^{\prime}$ we first note as above that

$$
\|g\|_{L_{t}^{1} L_{x}^{2}} \lesssim\|f\|_{L_{t}^{1} L_{x}^{2}}
$$

Now, according to (4.13), $w$ satisfies

$$
w_{t}-w_{x x x}+w_{x x}+w=g .
$$

Taking the $L_{x}^{2}$-scalar product with $w$ and using Cauchy-Schwarz yields

$$
\frac{1}{2} \frac{d}{d t}\|w\|_{L_{x}^{2}}^{2}-\left\|w_{x}\right\|_{L_{x}^{2}}^{2}+\|w\|_{L_{x}^{2}}^{2} \geq-\|g\|_{L_{x}^{2}}\|w\|_{L_{x}^{2}} .
$$

By the frequencies' localization of $w$ and the Bernstein inequality, $\left\|w_{x}\right\|_{L_{x}^{2}} \geq$ $\frac{1}{2} N\|w\|_{L_{x}^{2}}$. Therefore, for $t>0$ such that $\|w(t)\|_{L_{x}^{2}} \neq 0$, we can divide (4.16) by $\|w(t)\|_{L_{x}^{2}}$ to get

$$
N^{2}\|w(t)\|_{L_{x}^{2}} \lesssim \frac{d}{d t}\|w(t)\|_{L_{x}^{2}}+\|w(t)\|_{L_{x}^{2}}+\|g(t)\|_{L_{x}^{2}} .
$$


On the other hand, for $t>0$, the smoothness and non-negativity of $t \mapsto\|w(t)\|_{L_{x}^{2}}$ forces $\frac{d}{d t}\|w(t)\|_{L_{x}^{2}}^{2}=0$ as soon as $\|w(t)\|_{L_{x}^{2}}=0$. This ensures that (4.17) is actually valid for all $t>0$. Therefore integrating (4.17) on $] 0, t[$ we infer that

$$
\left\|w_{x x}\right\|_{L_{t}^{1} L_{x}^{2}} \sim N^{2}\|w\|_{L_{t}^{1} L_{x}^{2}} \lesssim\|w\|_{L_{t}^{\infty} L_{x}^{2}}+\|w\|_{L_{t}^{1} L_{x}^{2}}+\|g\|_{L_{t}^{1} L_{x}^{2}} .
$$

Since obviously,

$$
\|w\|_{L_{t}^{1} L_{x}^{2}}+\|w\|_{L_{t}^{\infty} L_{x}^{2}} \lesssim \sup _{t} \int_{0}^{\infty}\left\|e^{i\left(t-t^{\prime}\right) \xi^{3}} e^{-\left|t+t^{\prime}\right| \xi^{2}} \widehat{P_{N} f}\left(t^{\prime}\right)\right\|_{L_{\xi}^{2}} d t^{\prime} \lesssim\left\|P_{N} f\right\|_{L_{t}^{1} L_{x}^{2}}
$$

it follows that

$$
\left\|w_{x x}\right\|_{L_{t}^{1} L_{x}^{2}} \lesssim\left\|P_{N} f\right\|_{L_{t}^{1} L_{x}^{2}}
$$

which concludes the proof together with (4.14) and (4.15).

\section{Bilinear estimate}

In this section we provide a proof of the following crucial bilinear estimate.

Proposition 5.1. For all $u, v \in \mathcal{S}^{-1}$, we have

$$
\left\|\partial_{x}(u v)\right\|_{\mathcal{N}^{-1}} \lesssim\|u\|_{\mathcal{S}^{-1}}\|v\|_{\mathcal{S}^{-1}}
$$

We first remark that because of the $L_{\xi}^{2}$-structure of the spaces involved in our analysis we have the following localization property

$$
\|f\|_{\mathcal{S}^{-1}} \sim\left(\sum_{N}\left\|P_{N} f\right\|_{\mathcal{S}^{-1}}^{2}\right)^{1 / 2} \text { and }\|f\|_{\mathcal{N}^{-1}} \sim\left(\sum_{N}\left\|P_{N} f\right\|_{\mathcal{N}^{-1}}^{2}\right)^{1 / 2} .
$$

Performing a dyadic decomposition for $u, v$ we thus obtain

$$
\left\|\partial_{x}(u v)\right\|_{\mathcal{N}^{-1}} \sim\left(\sum_{N}\left\|\sum_{N_{1}, N_{2}} P_{N} \partial_{x}\left(P_{N_{1}} u P_{N_{2}} v\right)\right\|_{\mathcal{N}^{-1}}^{2}\right)^{1 / 2} .
$$

We can now reduce the number of cases to analyze by noticing that the right-hand side vanishes unless one of the following cases holds:

- (high-low interaction) $N \sim N_{2}$ and $N_{1} \lesssim N$,

- (low-high interaction) $N \sim N_{1}$ and $N_{2} \lesssim N$,

- (high-high interaction) $N \ll N_{1} \sim N_{2}$. 
The first and the second case are symmetric. In the first case, we can rewrite the right-hand side of (5.2) as

$$
\left\|\partial_{x}(u v)\right\|_{\mathcal{N}^{-1}} \sim\left(\sum_{N}\left\|P_{N} \partial_{x}\left(P_{\lesssim N} u P_{N} v\right)\right\|_{\mathcal{N}^{-1}}^{2}\right)^{1 / 2},
$$

and it suffices to prove the high-low estimate

$$
\left\|P_{N} \partial_{x}\left(P_{\lesssim N} u P_{N} v\right)\right\|_{\mathcal{N}^{-1}} \lesssim\|u\|_{\mathcal{S}^{-1}}\left\|P_{N} v\right\|_{\mathcal{S}^{-1}}
$$

for any dyadic $N$. If we consider now the third case, we easily get

$$
\left\|\partial_{x}(u v)\right\|_{\mathcal{N}^{-1}} \lesssim \sum_{N_{1}}\left\|P_{\ll N_{1}} \partial_{x}\left(P_{N_{1}} u P_{N_{1}} v\right)\right\|_{\mathcal{N}^{-1}},
$$

and it suffices to prove for any $N_{1}$ the high-high estimate

$$
\left\|P_{\ll N_{1}} \partial_{x}\left(P_{N_{1}} u P_{N_{1}} v\right)\right\|_{\mathcal{N}^{-1}} \lesssim\left\|P_{N_{1}} u\right\|_{\mathcal{S}^{-1}}\left\|P_{N_{1}} v\right\|_{\mathcal{S}^{-1}}
$$

since the claim follows then from the Cauchy-Schwarz inequality.

\subsection{Proof of (HL)}

We decompose the bilinear term as

$$
P_{N} \partial_{x}\left(P_{\lesssim N} u P_{N} v\right)=\sum_{N_{1} \lesssim N} \sum_{L, L_{1}, L_{2}} P_{N} Q_{L} \partial_{x}\left(P_{N_{1}} Q_{L_{1}} u P_{N} Q_{L_{2}} v\right) .
$$

Using the well-known resonance relation

$$
\xi_{1}^{3}+\xi_{2}^{3}+\xi_{3}^{3}=3 \xi_{1} \xi_{2} \xi_{3} \quad \text { whenever } \quad \xi_{1}+\xi_{2}+\xi_{3}=0,
$$

we see that non-trivial interactions only happen when

$$
L_{\max } \sim \max \left(N^{2} N_{1}, L_{\text {med }}\right)
$$

where $L_{\max } \geq L_{\text {med }} \geq L_{\text {min }}$ holds for $L, L_{1}, L_{2}$.

First we consider the easiest case $N_{1} \lesssim 1$. We take advantage of the $Y^{-1,-\frac{1}{2}}$ part of $\mathcal{N}^{-1}$ as well as of the Hölder and Bernstein inequalities to obtain

$$
\begin{aligned}
\sum_{N_{1} \lesssim 1}\left\|P_{N} \partial_{x}\left(P_{N_{1}} u P_{N} v\right)\right\|_{Y^{-1,-\frac{1}{2}}} & \lesssim \sum_{N_{1} \lesssim 1}\langle N\rangle^{-1} N\left\|P_{N}\left(P_{N_{1}} u P_{N} v\right)\right\|_{L_{t}^{1} L_{x}^{2}} \\
& \lesssim \sum_{N_{1} \lesssim 1}\left\|P_{N_{1}} u\right\|_{L_{t}^{2} L_{x}^{\infty}}\left\|P_{N} v\right\|_{L^{2}} \\
& \lesssim \sum_{N_{1} \lesssim 1} N_{1}^{1 / 2}\left\|P_{N_{1}} u\right\|_{L^{2}}\left\|P_{N} v\right\|_{L^{2}} \\
& \lesssim\|u\|_{\mathcal{S}^{-1}}\|v\|_{\mathcal{S}^{-1}}
\end{aligned}
$$

where we used (3.6) in the last estimate. One can now assume we have large space frequencies, i.e., $N \gtrsim N_{1} \gtrsim 1$. 
5.1.1. Case $L_{\max }=L$

In light of (5.4), we are in the region $L \gtrsim N^{2} N_{1}$. From the definition of $X^{-1, \frac{1}{2}, 1}$ we have

$$
\begin{aligned}
& \sum_{1 \lesssim N_{1} \lesssim N} \sum_{L \gtrsim N^{2} N_{1}}\left\|P_{N} Q_{L} \partial_{x}\left(P_{N_{1}} u P_{N} v\right)\right\|_{X^{-1,-\frac{1}{2}, 1}} \\
& \quad \lesssim \sum_{1 \lesssim N_{1} \lesssim N} \sum_{L \gtrsim N^{2} N_{1}} N^{-1}\langle L\rangle^{-1 / 2} N\left\|P_{N} Q_{L}\left(P_{N_{1}} u P_{N} v\right)\right\|_{L^{2}}
\end{aligned}
$$

Then, estimates (3.6) and (3.12) lead to the bound

$$
\begin{aligned}
& \lesssim \sum_{1 \lesssim N_{1} \lesssim N} N^{-1} N_{1}^{-1 / 2}\left\|P_{N_{1}} u\right\|_{L_{t}^{\infty} L_{x}^{2}}\left\|P_{N} v\right\|_{L_{t}^{2} L_{x}^{\infty}} \\
& \lesssim \sum_{1 \lesssim N_{1} \lesssim N} N_{1}^{1 / 2} N^{-1 / 2}\left\|P_{N_{1}} u\right\|_{L_{t}^{\infty} H_{x}^{-1}}\left\|P_{N} v\right\|_{L^{2}} \\
& \lesssim\|u\|_{\mathcal{S}^{-1}}\left\|P_{N} v\right\|_{\mathcal{S}^{-1}} .
\end{aligned}
$$

5.1.2. Case $L_{\max }=L_{1}$

Here, according to the resonance relation, we must have either $L_{1} \sim N^{2} N_{1}$ or $L_{1} \sim L_{\text {med }} \gtrsim N^{2} N_{1}$. Note that the latter case has been treated in Subsection 5.1.1 when $L_{\text {med }}=L$ and thus we can assume that $L_{\text {med }}=L_{2}$. The contribution for the former case can be estimated as follows:

$$
\begin{aligned}
& \sum_{1 \lesssim N_{1} \lesssim N} \sum_{L_{1} \sim N^{2} N_{1}}\left\|P_{N} \partial_{x}\left(P_{N_{1}} Q_{L_{1}} u P_{N} v\right)\right\|_{Y^{-1,-\frac{1}{2}}} \\
& \quad \lesssim \sum_{1 \lesssim N_{1} \lesssim N}\left\|P_{N_{1}} Q_{N^{2} N_{1}} u P_{N} v\right\|_{L_{t}^{1} L_{x}^{2}} \\
& \quad \lesssim \sum_{1 \lesssim N_{1} \lesssim N} N_{1}^{1 / 2}\left\|P_{N_{1}} Q_{N^{2} N_{1}} u\right\|_{L^{2}}\left\|P_{N} v\right\|_{L^{2}}
\end{aligned}
$$

Now we can exploit the fact that $L_{1} \sim N^{2} N_{1}$ to obtain

$$
N_{1}^{1 / 2}\left\|P_{N_{1}} Q_{N^{2} N_{1}} u\right\|_{L^{2}} \lesssim N^{-1} N_{1}\left(N_{1}^{-1} L_{1}^{1 / 2}\left\|P_{N_{1}} Q_{L_{1}} u\right\|_{L^{2}}\right),
$$

which combined with (3.6), (3.7) and Cauchy-Schwarz in $N_{1}$ yields the desired bound.

It remains to treat the case $L_{1} \sim L_{2} \gtrsim N^{2} N_{1}$. Arguing as before we get

$$
\begin{aligned}
& \sum_{1 \lesssim N_{1} \lesssim N} \sum_{L_{1} \sim L_{2} \gtrsim N^{2} N_{1}}\left\|P_{N} \partial_{x}\left(P_{N_{1}} Q_{L_{1}} u P_{N} Q_{L_{2}} v\right)\right\|_{Y^{-1,-\frac{1}{2}}} \\
& \quad \lesssim \sum_{1 \lesssim N_{1} \lesssim N} \sum_{L_{1} \gtrsim N^{2} N_{1}} N_{1}^{1 / 2}\left\|P_{N_{1}} Q_{L_{1}} u\right\|_{L^{2}}\left\|P_{N} Q_{L_{1}} v\right\|_{L^{2} .}
\end{aligned}
$$


In this region, (5.5) is still valid if we replace $Q_{N^{2} N_{1}}$ by $Q_{L_{1}}$. Applying this for $u$ and $v$, this provides the bound

$$
\begin{aligned}
& \lesssim \sum_{1 \lesssim N_{1} \lesssim N} N_{1}^{1 / 2} N^{-1}\left(\sum_{L_{1}}\left(N_{1}^{-1} L_{1}^{1 / 2}\left\|P_{N_{1}} Q_{L_{1}} u\right\|_{L^{2}}\right)^{2}\right)^{1 / 2} \\
& \quad \times\left(\sum_{L_{1}}\left(N^{-1} L_{1}^{1 / 2}\left\|P_{N} Q_{L_{1}} v\right\|_{L^{2}}\right)^{2}\right)^{1 / 2} \\
& \lesssim \sum_{1 \lesssim N_{1} \lesssim N} N_{1}^{1 / 2} N^{-1}\left\|P_{N_{1}} u\right\|_{\mathcal{S}^{-1}}\left\|P_{N} v\right\|_{\mathcal{S}^{-1}}
\end{aligned}
$$

which is acceptable (with about $N^{-1 / 2}$ of spare).

5.1.3. Case $L_{\max }=L_{2}$

By (5.4), it suffices to consider the case $L_{2} \sim N^{2} N_{1}$. With a similar argument we get

$$
\begin{aligned}
& \sum_{N_{1} \lesssim N} \sum_{L_{2} \sim N^{2} N_{1}}\left\|P_{N} \partial_{x}\left(P_{N_{1}} u P_{N} Q_{L_{2}} v\right)\right\|_{Y^{-1,-\frac{1}{2}}} \\
& \quad \lesssim \sum_{N_{1} \lesssim N} N_{1}^{1 / 2}\left\|P_{N_{1}} u\right\|_{L^{2}}\left\|P_{N} Q_{N^{2} N_{1}} v\right\|_{L^{2}} \\
& \lesssim\left(\sum_{N_{1}}\left\|P_{N_{1}} u\right\|_{L^{2}}^{2}\right)^{1 / 2}\left(\sum_{N_{1}}\left(N_{1}^{1 / 2}\left\|P_{N} Q_{N^{2} N_{1}} v\right\|_{L^{2}}\right)^{2}\right)^{1 / 2} \\
& \quad \lesssim\|u\|_{\mathcal{S}^{-1}}\left(\sum_{L_{2}}\left(N^{-1} L_{2}^{1 / 2}\left\|P_{N} Q_{L_{2}} v\right\|_{L^{2}}\right)^{2}\right)^{1 / 2}
\end{aligned}
$$

which achieves the proof of (HL).

\subsection{Proof of $(\mathrm{HH})$}

Performing the decomposition

$$
P_{\ll N_{1}} \partial_{x}\left(P_{N_{1}} u P_{N_{1}} v\right)=\sum_{N \ll N_{1}} \sum_{L, L_{1}, L_{2}} P_{N} Q_{L} \partial_{x}\left(P_{N_{1}} Q_{L_{1}} u P_{N_{1}} Q_{L_{2}} v\right),
$$

we see from (5.3) that we may restrict ourself to the region where

$$
L_{\max } \sim \max \left(N_{1}^{2} N, L_{\text {med }}\right) .
$$


Moreover, we may assume by symmetry that $L_{1} \geq L_{2}$. Low frequencies $N \lesssim 1$ are easily handled:

$$
\begin{aligned}
\sum_{N \lesssim 1}\left\|P_{N} \partial_{x}\left(P_{N_{1}} u P_{N_{1}} v\right)\right\|_{Y^{-1,-\frac{1}{2}}} & \lesssim \sum_{N \lesssim 1}\langle N\rangle^{-1} N\left\|P_{N}\left(P_{N_{1}} u P_{N_{1}} v\right)\right\|_{L_{t}^{1} L_{x}^{2}} \\
& \lesssim \sum_{N \lesssim 1}\left\|P_{N_{1}} u\right\|_{L^{2}}\left\|P_{N_{1}} v\right\|_{L^{2}} \\
& \lesssim\left\|P_{N_{1}} u\right\|_{\mathcal{S}^{-1}}\left\|P_{N_{1}} v\right\|_{\mathcal{S}^{-1}} .
\end{aligned}
$$

Therefore it is sufficient to consider $N_{1} \gg N \gtrsim 1$.

5.2.1. Case $L_{\max }=L$

In this region one has $L \gtrsim N_{1}^{2} N$. Let us assume $L_{1} \lesssim N_{1}^{2} N^{1-\varepsilon}$ for some $\varepsilon>0$, so that we wish to bound

$$
\left\|\sum_{1 \lesssim N \ll N_{1}} P_{N} Q_{\gtrsim N_{1}^{2} N} \partial_{x}\left(P_{N_{1}} Q_{\lesssim N_{1}^{2} N^{1-\varepsilon}} u P_{N_{1}} v\right)\right\|_{X^{-1,-\frac{1}{2}, 1}} .
$$

According to the triangle inequality, it suffices to estimate

$$
\sum_{1 \lesssim N \ll N_{1}} \sum_{\substack{L \gtrsim N_{1}^{2} N \\ L_{1} \lesssim N_{1}^{2} N^{1-\varepsilon}}} L^{-1 / 2}\left\|P_{N_{1}} Q_{L_{1}} u P_{N_{1}} v\right\|_{L^{2}} .
$$

In order to get a suitable control for this term, we use the Kato smoothing effect (3.13) together with estimate (3.6) to get

$$
\begin{aligned}
\left\|P_{N_{1}} Q_{L_{1}} u P_{N_{1}} v\right\|_{L^{2}} & \lesssim\left\|P_{N_{1}} Q_{L_{1}} u\right\|_{L_{x}^{2} L_{t}^{\infty}}\left\|P_{N_{1}} v\right\|_{L_{x}^{\infty} L_{t}^{2}} \\
& \lesssim L_{1}^{1 / 2}\left\|P_{N_{1}} u\right\|_{\mathcal{S}^{-1}}\left\|P_{N_{1}} v\right\|_{\mathcal{S}^{-1}} .
\end{aligned}
$$

Therefore it remains to establish

$$
\sum_{1 \lesssim N \ll N_{1}} \sum_{\substack{L \gtrsim N_{1}^{2} N \\ L 1 \lesssim N_{1}^{2} N^{1-\varepsilon}}} L^{-1 / 2} L_{1}^{1 / 2} \lesssim 1,
$$

but this is easily verified by Schur's test for any $\varepsilon>0$. The situation where $L_{2} \lesssim$ $N_{1}^{2} N^{1-\varepsilon}$ is identical to the previous one and we suppose now $L_{1}, L_{2} \gtrsim N_{1}^{2} N^{1-\widetilde{\varepsilon}}$. Estimating the $\mathcal{N}^{-1}$-norm by the $Y^{-1,-\frac{1}{2}}$-norm, and using the Hölder and Bernstein 
inequalities we see that the contribution in this case is bounded by

$$
\begin{aligned}
& \sum_{1 \lesssim N \ll N_{1}} \| P_{N}\left(P_{N_{1}} Q_{\gtrsim N_{1}^{2} N^{1-\varepsilon}} u P_{N_{1}} Q_{\gtrsim N_{1}^{2} N^{1-\varepsilon}} v \|_{L_{t}^{1} L_{x}^{2}}\right. \\
& \quad \lesssim \sum_{1 \lesssim N \ll N_{1}} N^{1 / 2}\left\|P_{N_{1}} Q_{\gtrsim N_{1}^{2} N^{1-\varepsilon}} u\right\|_{L^{2}}\left\|P_{N_{1}} Q_{\gtrsim N_{1}^{2} N^{1-\varepsilon}} v\right\|_{L^{2}} .
\end{aligned}
$$

On the other hand the resonance relation and (3.7) yield

$$
\begin{aligned}
N^{1 / 2}\left\|P_{N_{1}} Q_{\gtrsim N_{1}^{2} N^{1-\varepsilon}} u\right\|_{L^{2}} & \lesssim N^{\varepsilon / 2}\left(\sum_{L_{1}}\left[N_{1}^{-1} L_{1}^{1 / 2}\left\|P_{N_{1}} Q_{L_{1}} u\right\|_{L^{2}}\right]^{2}\right)^{1 / 2} \\
& \lesssim N^{\varepsilon / 2}\left\|P_{N_{1}} u\right\|_{\mathcal{S}^{-1}}
\end{aligned}
$$

and similarly for $v$. Inserting this into (5.8) we deduce

$$
(5.8) \lesssim \sum_{N \gtrsim 1} N^{-1 / 2+\varepsilon}\left\|P_{N_{1}} u\right\|_{\mathcal{S}^{-1}}\left\|P_{N_{1}} v\right\|_{\mathcal{S}^{-1}}
$$

which is acceptable for $\varepsilon<1 / 2$.

5.2.2. Case $L_{\max }=L_{1}$

First we consider the region $L_{1} \sim N_{1}^{2} N$ and we want to estimate

$$
\begin{aligned}
& \left\|\sum_{N \ll N_{1}} P_{N} \partial_{x}\left(P_{N_{1}} Q_{N_{1}^{2} N} u P_{N_{1}} v\right)\right\|_{Y^{-1,-\frac{1}{2}}} \\
& \quad \lesssim\left(\sum_{N}\left[N^{1 / 2}\left\|P_{N_{1}} Q_{N_{1}^{2} N} u\right\|_{L^{2}}\left\|P_{N_{1}} v\right\|_{L^{2}}\right]^{2}\right)^{1 / 2}
\end{aligned}
$$

where we took care of not using the triangle inequality in order to keep the $\ell^{2}$-norm in $N$. The term $\left\|P_{N_{1}} u\right\|_{L^{2}}$ can be handled with the help of (3.6), while the change of variable $N \sim L_{1} N_{1}^{-2}$ for fixed $N_{1}$ leads to the bound

$$
\lesssim\left(\sum_{L_{1}}\left[N_{1}^{-1} L_{1}^{1 / 2}\left\|P_{N_{1}} Q_{L_{1}} u\right\|_{L^{2}}\right]^{2}\right)^{1 / 2}\left\|P_{N_{1}} v\right\|_{\mathcal{S}^{-1}} \lesssim\left\|P_{N_{1}} u\right\|_{\mathcal{S}^{-1}}\left\|P_{N_{1}} v\right\|_{\mathcal{S}^{-1}}
$$


Finally in the case $L_{1} \sim L_{2} \gtrsim N_{1}^{2} N$, arguing as in Subsection 5.1.2, we get

$$
\begin{aligned}
& \left\|\sum_{1 \lesssim N \ll N_{1}} \sum_{L_{1} \sim L_{2} \gg N_{1}^{2} N} P_{N} \partial_{x}\left(P_{N_{1}} Q_{L_{1}} u P_{N_{1}} Q_{L_{2}} v\right)\right\|_{Y^{-1,-\frac{1}{2}}} \\
& \lesssim \sum_{1 \lesssim N \ll N_{1}} \sum_{L_{1} \gg N_{1}^{2} N} N^{1 / 2}\left\|P_{N_{1}} Q_{L_{1}} u\right\|_{L^{2}}\left\|P_{N_{1}} Q_{L_{1}} v\right\|_{L^{2}} \\
& \lesssim \sum_{N \gtrsim 1} N^{-1 / 2}\left(\sum_{L_{1}}\left(N_{1}^{-1} L_{1}^{1 / 2}\left\|P_{N_{1}} Q_{L_{1}} u\right\|_{L^{2}}\right)^{2}\right)^{1 / 2} \\
& \quad \times\left(\sum_{L_{1}}\left(N_{1}^{-1} L_{1}^{1 / 2}\left\|P_{N_{1}} Q_{L_{1}} v\right\|_{L^{2}}\right)^{2}\right)^{1 / 2},
\end{aligned}
$$

which is acceptable (with about $N^{-1 / 2}$ of spare).

\section{Well-posedness}

In this section we prove the well-posedness result. Using a standard fixed-point procedure, it is clear that the bilinear estimate (5.1) allows us to show local wellposedness but for small initial data only. This is because $H^{-1}$ appears as a critical space for KdV-B and thus we cannot get the desired contraction factor in our estimates. In order to remove the size restriction on the data, we need to change the metric on our resolution space.

For $\beta \geq 1$, let us define the following norm on $\mathcal{S}^{-1}$,

$$
\|u\|_{\mathcal{Z}_{\beta}}=\inf _{\substack{u=u_{1}+u_{2} \\ u_{1} \in \mathcal{S}^{-1}, u_{2} \in \mathcal{S}^{0}}}\left\{\left\|u_{1}\right\|_{\mathcal{S}^{-1}}+\frac{1}{\beta}\left\|u_{2}\right\|_{\mathcal{S}^{0}}\right\} .
$$

Note that this norm is equivalent to $\|\cdot\|_{\mathcal{S}^{-1}}$. Now we will need the following modification of Proposition 5.1. This new proposition means that as soon as we assume more regularity on $u$ we can get a contractive factor for small times in the bilinear estimate.

Proposition 6.1. There exists $v>0$ such that for all $(u, v) \in \mathcal{S}^{0} \times \mathcal{S}^{-1}$, with compact support (in time) in $[-T, T]$, it holds

$$
\left\|\partial_{x}(u v)\right\|_{\mathcal{N}^{-1}} \lesssim T^{v}\|u\|_{\mathcal{S}^{0}}\|v\|_{\mathcal{S}^{-1}}
$$

Proof. It suffices to slightly modify the proof of Proposition 5.1 to make use of the following result that can be found in [9, Lemma 3.1] (see also [16, Lemma 3.6]): 
For any $\theta>0$, there exists $\mu=\mu(\theta)>0$ such that for any smooth function $f$ with compact support in time in $[-T, T]$,

$$
\left\|\mathcal{F}_{t, x}^{-1}\left(\frac{\hat{f}(\tau, \xi)}{\left\langle\tau-\xi^{3}\right\rangle^{\theta}}\right)\right\|_{L_{t, x}^{2}} \lesssim T^{\mu}\|f\|_{L_{t, x}^{2,2}} .
$$

According to (3.7) this ensures, in particular, that for any $w \in \mathcal{S}^{0}$ with compact support in $[-T, T]$ it holds

$$
\|w\|_{L_{t}^{2} H^{3 / 4}} \lesssim\|w\|_{X^{0,3 / 8,2}} \lesssim T^{\mu\left(\frac{1}{8}\right)}\|w\|_{X^{0,1 / 2,2}} \lesssim T^{\mu\left(\frac{1}{8}\right)}\|w\|_{\mathcal{S}^{0}}
$$

It is pretty clear that the interactions between high frequencies of $u$ and high or low frequencies of $v$ can be treated following the proof of Proposition 5.1 and using (6.3). The region that seems the most dangerous is that of interactions between low frequencies of $u$ and high frequencies of $v$, that is the region of $(H L)$ in the proof of Proposition 5.1. But actually this region can also be easily treated. For instance in the case 5.1.1 it suffices to notice that

$$
\begin{aligned}
\sum_{1 \lesssim N_{1} \lesssim N} & \sum_{L \gtrsim N^{2} N_{1}}\left\|P_{N} Q_{L} \partial_{x}\left(P_{N_{1}} u P_{N} v\right)\right\|_{X^{-1,-\frac{1}{2}, 1}} \\
& \lesssim \sum_{1 \lesssim N_{1} \lesssim N} \sum_{L \gtrsim N^{2} N_{1}} N^{-1}\langle L\rangle^{-1 / 2} N\left\|P_{N} Q_{L}\left(P_{N_{1}} u P_{N} v\right)\right\|_{L^{2}} \\
& \lesssim \sum_{1 \lesssim N_{1} \lesssim N} N^{-1} N_{1}^{-1 / 2}\left\|P_{N_{1}} u\right\|_{L_{t}^{2} L_{x}^{\infty}}\left\|P_{N} v\right\|_{L_{t}^{\infty} L_{x}^{2}} \\
& \lesssim \sum_{1 \lesssim N_{1} \lesssim N} N_{1}^{-1 / 2}\left\|P_{N_{1}} u\right\|_{L_{t}^{2} H_{x}^{1 / 2}\left\|P_{N} v\right\|_{L_{t}^{\infty} H_{x}^{-1}}} \\
& \lesssim T
\end{aligned}
$$

and in the case 5.1.2 it simply suffices to replace (5.5) by

$$
N_{1}^{1 / 2}\left\|P_{N_{1}} Q_{N^{2} N_{1}} u\right\|_{L^{2}} \lesssim N_{1}^{-1 / 4}\left\|P_{N_{1}} u\right\|_{L_{t}^{2} H_{x}^{3 / 4}} \lesssim N_{1}^{-1 / 4} T^{\mu\left(\frac{1}{8}\right)}\|u\|_{\mathcal{S}^{0}} .
$$

The other cases can be handled in a similar way.

We are now in a position to prove that the application

$$
F_{\phi}^{T}: u \mapsto \eta(t)\left[W(t) \phi-\frac{1}{2} \mathcal{L} \partial_{x}\left(\eta_{T} u\right)^{2}\right],
$$


where $\mathcal{L}$ is defined in (4.8), is contractive on a ball of $\mathcal{Z}_{\beta}$ for suitable $\beta>0$ and $T>0$ small enough. Assuming this for a while, the local part of Theorem 1.1 follows using standard arguments. Note that the uniqueness will hold in the restriction spaces $\mathcal{S}_{\tau}^{-1}$ endowed with the norm

$$
\|u\|_{\mathcal{S}_{\tau}^{-1}}:=\inf _{v \in \mathcal{S}^{-1}}\left\{\|v\|_{\mathcal{S}^{-1}}, v \equiv u \text { on }\right] 0, \tau[\} .
$$

Finally, to see that the solution $u$ can be extended for all positive times and belongs to $C\left(\mathbb{R}_{+}^{*} ; H^{\infty}\right)$ it suffices to notice that, according to (3.6), $u \in \mathcal{S}_{\tau}^{-1} \hookrightarrow$ $L^{2}(] 0, \tau[\times \mathbb{R})$. Therefore, for any $0<\tau^{\prime}<\tau$ there exists $\left.t_{0} \in\right] 0, \tau^{\prime}$ [, such that $u\left(t_{0}\right)$ belongs to $L^{2}(\mathbb{R})$. Since according to [16], (1.1) is globally well-posed in $L^{2}(\mathbb{R})$ with a solution belonging to $C\left(\mathbb{R}_{+}^{*} ; H^{\infty}(\mathbb{R})\right)$, the conclusion follows.

In order to prove that $F_{\phi}^{T}$ is contractive, the first step is to establish the following result.

Proposition 6.2. For any $\beta \geq 1$ there exists $0<T=T(\beta)<1$ such that for any $u, v \in \mathcal{Z}_{\beta}$ with compact support in $[-T, T]$ we have

$$
\left\|\mathcal{L} \partial_{x}(u v)\right\|_{\mathcal{Z}_{\beta}} \lesssim\|u\|_{\mathcal{Z}_{\beta}}\|v\|_{\mathcal{Z}_{\beta}}
$$

Assume for the moment that (6.4) holds and let $u_{0} \in H^{-1}$ and $\alpha>0$. Split the data $u_{0}$ into low and high frequencies:

$$
u_{0}=P_{\lesssim N} u_{0}+P_{\gg N} u_{0}
$$

for a dyadic number $N$. Taking $N=N(\alpha)$ large enough, it is obvious to check that $\left\|P_{\gg N} u_{0}\right\|_{H^{-1}} \leq \alpha$. Hence, according to (4.1),

$$
\left\|\eta(\cdot) W(\cdot) P_{\gg N} u_{0}\right\|_{\mathcal{Z}_{\beta}} \lesssim \alpha .
$$

Using now the $\mathcal{S}^{0}$-part of $\mathcal{Z}_{\beta}$, we control the low frequencies as follows:

$$
\left\|\eta(\cdot) W(\cdot) P_{\lesssim_{N}} u_{0}\right\|_{\mathcal{S}^{0}} \lesssim \frac{1}{\beta}\left\|P_{\lesssim N} u_{0}\right\|_{L^{2}} \lesssim \frac{N}{\beta}\left\|u_{0}\right\|_{H^{-1}} .
$$

Thus we get

$$
\left\|\eta(\cdot) W(\cdot) P_{\lesssim N} u_{0}\right\|_{\mathcal{Z}_{\beta}} \lesssim \alpha \text { for } \beta \gtrsim \frac{N\left\|u_{0}\right\|_{H^{-1}}}{\alpha}
$$

Since $\alpha$ can be chosen as small as needed, we conclude with (6.4) that $F_{\phi}^{T}$ is contractive on a ball of $\mathcal{Z}_{\beta}$ of radius $R \sim \alpha$ as soon as $\beta \gtrsim N\left\|u_{0}\right\|_{H^{-1}} / \alpha$ and $T=T(\beta)$. 
Proof of Proposition 6.2. By definition, on the function space $\mathcal{Z}_{\beta}$ there exist $u_{1}$, $v_{1} \in \mathcal{S}^{-1}$ and $u_{2}, v_{2} \in \mathcal{S}^{0}$ such that $u=u_{1}+v_{1}, v=v_{1}+v_{2}$ and

$$
\begin{aligned}
\left\|u_{1}\right\|_{\mathcal{S}^{-1}}+\frac{1}{\beta}\left\|u_{2}\right\|_{\mathcal{S}^{0}} & \leq 2\|u\|_{\mathcal{Z}_{\beta}}, \\
\left\|v_{1}\right\|_{\mathcal{S}^{-1}}+\frac{1}{\beta}\left\|v_{2}\right\|_{\mathcal{S}^{0}} & \leq 2\|v\|_{\mathcal{Z}_{\beta}} .
\end{aligned}
$$

Thus one can decompose the left-hand side of (6.4) as

$$
\begin{aligned}
\left\|\mathcal{L} \partial_{x}(u v)\right\|_{\mathcal{Z}_{\beta}} & \lesssim\left\|\mathcal{L} \partial_{x}\left(u_{1} v_{1}\right)\right\|_{\mathcal{S}^{-1}}+\left\|\mathcal{L} \partial_{x}\left(u_{1} v_{2}+u_{2} v_{1}\right)\right\|_{\mathcal{S}^{-1}}+\left\|\mathcal{L} \partial_{x}\left(u_{2} v_{2}\right)\right\|_{\mathcal{S}^{-1}} \\
& =I+I I+I I I .
\end{aligned}
$$

From the estimates (4.9) and (5.1) we get

$$
I \lesssim\left\|\partial_{x}\left(u_{1} v_{1}\right)\right\|_{\mathcal{N}^{-1}} \lesssim\left\|u_{1}\right\|_{S^{-1}}\left\|v_{1}\right\|_{\mathcal{S}^{-1}} \lesssim\|u\|_{\mathcal{Z}_{\beta}}\|v\|_{\mathcal{Z}_{\beta}} .
$$

On the other hand, we obtain from (6.1) that

$$
I I I \lesssim T^{v}\left\|u_{2}\right\|_{\mathcal{S}^{0}}\left\|v_{2}\right\|_{\mathcal{S}^{0}} \lesssim \beta^{2} T^{v}\|u\|_{\mathcal{Z}_{\beta}}\|v\|_{\mathcal{Z}_{\beta}}
$$

and

$$
\begin{aligned}
I I & \lesssim T^{v}\left(\left\|u_{1}\right\|_{\mathcal{S}^{-1}}\left\|v_{2}\right\|_{\mathcal{S}^{0}}+\left\|u_{2}\right\|_{\mathcal{S}^{0}}\left\|v_{1}\right\|_{\mathcal{S}^{-1}}\right) \\
& \lesssim \beta T^{v}\|u\|_{\mathcal{Z}_{\beta}}\|v\|_{\mathcal{Z}_{\beta}} .
\end{aligned}
$$

We thus get

$$
\left\|\mathcal{L} \partial_{x}(u v)\right\|_{\mathcal{Z}_{\beta}} \lesssim\left(1+\left(\beta+\beta^{2}\right) T^{v}\right)\|u\|_{\mathcal{Z}_{\beta}}\|v\|_{\mathcal{Z}_{\beta}} .
$$

This ensures that (6.4) holds for $T \sim \beta^{-2 / \nu} \leq 1$.

\section{References}

[1] D. BEKIRANOv, The initial-value problem for the generalized Burgers' equation, Differential Integral Equations 9 (6) (1996), 1253-1265.

[2] I. BEJENARU and T. TAO, Sharp well-posedness and ill-posedness results for a quadratic non-linear Schrödinger equation, J. Funct. Anal. 233 (2006), 228-259.

[3] J. BOURGAIN, Fourier transform restriction phenomena for certain lattice subsets and application to nonlinear evolution equations. II. The KdV equation, Geom. Funct. Anal. 3 (1993), 209-262.

[4] J. Bourgain, Periodic Korteveg de Vries equation with measures as initial data, Selecta Math. 3 (1993), 115-159.

[5] M. Christ, J. Colliander and T. TAO, Asymptotics, frequency modulation, and low regularity ill-posedness for canonical defocusing equations, Amer. J. Math. 125 (2003), $1235-1293$. 
[6] D. B. DIX, Nonuniqueness and uniqueness in the initial-value problem for Burger's equation, SIAM J. Math. Anal. 27 (1996), 708-724.

[7] P. GÉRARD, Nonlinear Schrödinger equations in inhomogeneous media: wellposedness and illposedness of the Cauchy problem, In: "International Congress of Mathematicians", Vol. III, Eur. Math. Soc., Zürich, 2006, 157-182.

[8] J. GINIBRE, Le problème de Cauchy pour des EDP semi-linéaires périodiques en variables d'espace (d'après Bourgain), In: "Séminaire Bourbaki 796", Astérique 237, 1995, 163-187.

[9] J. GinibRe, Y. Tsutsumi and G. Velo, On the Cauchy problem for the Zakharov system, J. Funct. Anal. 133 (1997), 50-68.

[10] Z. Guo, Global Well-posedness of Korteweg-de Vries equation in $H^{-3 / 4}(\mathbb{R})$, J. Math. Pures Appl. 91 (2009), 583-597.

[11] Z. GUO and B. WANG, Global well-posedness and inviscid limit for the Korteweg-de VriesBurgers equation, J. Differential Equations 246 (2009), 3864-3901.

[12] T. KAPPELER and P. Topalov, Global wellposedness of $K d V$ in $H^{-1}(\mathbb{T}, \mathbb{R})$, Duke Math. J. 135 (2006), 327-360.

[13] C. E. Kenig, G. Ponce and L. Vega, A bilinear estimate with applications to the KdV equation, J. Amer. Math. Soc. 9 (1996), 573-603.

[14] N. Kishimoto, Well-posedness of the Cauchy problem for the Korteweg-de Vries equation at the critical regularity, Differential Integral Equations 22 (2009), 447-464.

[15] L. Molinet and S. Vento, Sharp ill-posedness and well-posedness results for the KdVBurgers equation: the periodic case, Trans. Amer. Math. Soc., to appear.

[16] L. Molinet and F. RiBAUd, On the low regularity of the Korteweg-de Vries-Burgers equation, Int. Math. Res. Not. 37 (2002), 1979-2005.

[17] E. OTT and N. SudAn, Damping of solitary waves, Phys. Fluids 13 (1970), 1432-1434.

[18] T. TAO, Multilinear weighted convolution of $L^{2}$-functions, and applications to nonlinear dispersive equations, Amer. J. Math. 123 (2001), 839-908.

[19] T. TAO, Scattering for the quartic generalised Korteweg-de Vries equations, J. Differential Equations 232 (2007), 623-651.

[20] D. TATARU, On global existence and scattering for the wave maps equation, Amer. J. Math. 123 (2001), 37-77.

Laboratoire de Mathématiques et Physique Théorique Université François Rabelais Tours Fédération Denis Poisson-CNRS Parc Grandmont, 37200 Tours, France Luc.Molinet@lmpt.univ-tours.fr

L.A.G.A., Institut Galilée

Université Paris 13

93430 Villetaneuse, France

vento@math.univ-paris13.fr 